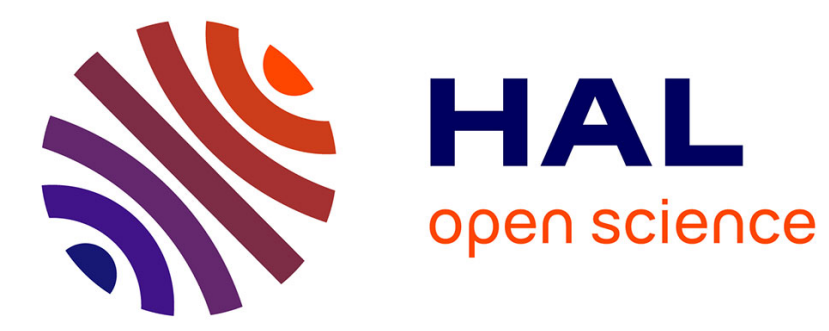

\title{
Tidally Heated Convection and the Occurrence of Melting in Icy Satellites: Application to Europa
}

K Vilella, G Choblet, W.-E Tsao, F Deschamps

\section{To cite this version:}

K Vilella, G Choblet, W.-E Tsao, F Deschamps. Tidally Heated Convection and the Occurrence of Melting in Icy Satellites: Application to Europa. Journal of Geophysical Research. Planets, 2020, 125 (3), pp.e2019JE006248. 10.1029/2019je006248 . hal-03420850

\section{HAL Id: hal-03420850 https://hal.science/hal-03420850}

Submitted on 9 Nov 2021

HAL is a multi-disciplinary open access archive for the deposit and dissemination of scientific research documents, whether they are published or not. The documents may come from teaching and research institutions in France or abroad, or from public or private research centers.
L'archive ouverte pluridisciplinaire HAL, est destinée au dépôt et à la diffusion de documents scientifiques de niveau recherche, publiés ou non, émanant des établissements d'enseignement et de recherche français ou étrangers, des laboratoires publics ou privés. 


\section{JGR Planets}

\section{RESEARCH ARTICLE \\ 10.1029/2019JE006248 \\ Tidally Heated Convection and the Occurrence of Melting in Icy Satellites: Application to Europa}

Key Points:

- For Europa, isolated melt pockets can only be generated for a specific range of the ice layer thickness (15-35 km)

- For tidal heating rates exceeding a threshold, the surface heat flux remains constant even with increasing amount of tidal heating

- We propose a reappraisal of Europa's thermal state that, when extrapolated, suggests a very thick ice shell $(50-90 \mathrm{~km})$

Supporting Information:

- Supporting Information S1

Correspondence to:

K. Vilella and and F. Deschamps,

kennyvilella@gmail.com;

frederic@earth.sinica.edu.tw

Citation:

Vilella, K., Choblet, G., Tsao, W.-E., \& Deschamps, F. (2020). Tidally heated convection and the occurrence of melting in icy satellites: Application to Europa. Journal of Geophysical

Research: Planets, 125, e2019JE006248. https://doi.org/10.1029/2019JE006248

Received 22 OCT 2019

Accepted 21 FEB 2020

Accepted article online 9 FEB 2020

(C)2020. American Geophysical Union. All Rights Reserved.

\author{
K. Vilella ${ }^{1,2}$ (D) G. Choblet ${ }^{3}\left(\mathbb{D}\right.$, W.-E. Tsao' ${ }^{1}$, and F. Deschamps ${ }^{1}$ (iD \\ ${ }^{1}$ Institute of Earth Sciences, Academia Sinica, Taipei, Taiwan, ${ }^{2}$ JSPS International Research Fellow, Hokkaido University, \\ Sapporo, Japan, ${ }^{3}$ Laboratoire de Planétologie et Géodynamique, UMR-CNRS 6112, Université de Nantes, Nantes, France
}

\begin{abstract}
Observations of icy satellites have revealed widespread marks of cryovolcanism. Because aqueous cryomagmas are negatively buoyant, two processes are required to explain these observations: one mechanism to generate melt close enough to the surface and another one to transport this melt to the surface. Here, we investigate the generation of melting in a systematic way, using a set of 85 numerical simulations where we vary the viscosity contrast, Rayleigh number, and tidal heating rate. Applied to Europa, considering a hydrosphere composed of pure water, our simulations suggest that isolated melt pockets can be generated close to the surface $(\sim 5 \mathrm{~km})$ as long as the ice layer thickness $\left(d^{*}\right)$ remains modest $\left(15 \leq d^{*} \leq 35 \mathrm{~km}\right)$. However, the generation of melting becomes increasingly difficult as the amount of antifreeze compounds in the subsurface ocean increases. Furthermore, the proportion of melting increases very sharply with increasing tidal heating rate. In particular, when the tidal heating rate exceeds a threshold, an asymptotic regime is reached where the surface heat flux remains constant indicating that the tidal heat generated above this threshold is only used for melting the ice shell. In that regime, we found a direct relationship between the surface heat flux and $d^{*}$. Finally, we provide a new assessment of Europa's thermal state. Based on available constraints, we propose that the ice shell thickness should exceed $15 \mathrm{~km}$. However, $d^{*} \sim 15-35 \mathrm{~km}$ implies a tidal power $(>2 \mathrm{TW})$ much larger than expected. An extrapolation of the trends suggested by our results indicates that a more reasonable tidal power $(\sim 1 \mathrm{TW})$ would involve $d^{*} \sim 50-90 \mathrm{~km}$.
\end{abstract}

Plain Language Summary Eruptions of cryomagmas have been inferred in many icy satellites. In many cases, the mechanisms producing these eruptions are not yet unambiguously identified. Here, we focus on one part of the problem, which is how to generate melting within the ice shell of icy satellites. To do so, we conducte a large number of numerical simulations in 3-D. When applied to Europa, our results indicate that melt reservoirs may only be generated for a moderately thick ice shell. We also find that, if a large amount of melting is generated, the surface heat flux is a good proxy for the thickness of the ice shell. Finally, we provide a new assessment of Europa's thermal state. An extrapolation of our results, combined to properties generally assumed for Europa, suggests a thick ice shell ( $\sim 50-90 \mathrm{~km})$.

\section{Introduction}

Data gathered from flybys of icy moons by various spacecrafts during the past four decades have led to the conclusion that cryovolcanism is likely a common feature of these bodies (Kargel, 1995). Evidence of ancient volcanic activity has been observed on Ganymede (Schenk et al., 2001), Miranda (Pappalardo et al., 1997; Schenk, 1991), Ariel (Schenk, 1991), while current or contemporary activity has been suggested on Enceladus (Hansen et al., 2006; Porco et al., 2006; Spencer et al., 2006), Europa (Sparks et al., 2017; Jia et al., 2018; Roth et al., 2014), Titan (Elachi et al., 2005; Lopes et al., 2007, 2013) and Triton (Smith et al., 1989). In contrast to Earth-like magma, aqueous cryomagmas are negatively buoyant with respect to ice. Consequently, their eruption at the surface of icy bodies cannot be due to buoyancy-driven upwelling, making the explanation of the observations more challenging.

Several mechanisms have been proposed to overcome this difficulty. For instance, the transport of melt to the surface may be triggered by fractional crystallization (Mitri et al., 2008) or pressurization (Lesage et al., 2020; Showman \& Han, 2004). Importantly, and regardless of the mechanism considered, the presence of liquid reservoirs close to the surface is required. While there is, to date, no direct observation of melt reservoirs, their presence may be needed to explain surface tectonic features such as double ridges on Europa 
(Dombard et al., 2013). The presence of these melt reservoirs does not necessarily imply cryovolcanism, which may, in addition, depend on the location and amount of this melt. For instance, melting located too deep or available in too small quantity may not allow the occurrence of cryovolcanism. By contrast, melting generated in a significant amount and close enough to the surface may induce cryovolcanism or at least produce distinctive surface features. Note that, for these melt reservoirs, the minimum depth to generate surface features is unclear. Fractional crystallization mechanisms may involve depths up to $10 \mathrm{~km}$ (Mitri et al., 2008), while pressurization mechanisms require shallower reservoirs (typically depths at which ice behaves as an elastic medium, see, for instance, Lesage et al., 2020; Michaut \& Manga, 2014; Manga \& Michaut, 2017) in agreement with observations (e.g., Schmidt et al., 2011).

Nevertheless, the mechanism at the origin of these reservoirs remains a subject of debate. For Europa, a possible hypothesis is that tidal motion produces strike-slip displacements associated with shear heating and melting (Gaidos \& Nimmo, 2000; Nimmo \& Gaidos, 2002). Alternatively, melting may be produced by convective currents sustained by tidal heating (Kalousová et al., 2014; Sotin et al., 2002). Tidally heated convection in icy satellites has been widely studied with numerical simulations (e.g., Allu Peddinti \& McNamara, 2019; Běhounková et al., 2010; Han \& Showman, 2010; Roberts \& Nimmo, 2008; Tobie et al., 2003). Interestingly, these studies have found that the important characteristics of the convective flow (e.g., heat flow and temperature distribution) are sensitive to a number of parameters, especially the thickness of the ice layer, the tidal heating rate, and the ice viscosity. For a few values of these parameters, Han and Showman (2010) found that melt reservoirs could be generated in Europa. The values of these parameters are however poorly constrained for most icy satellites.

Here, we propose to follow an alternative strategy based on a systematic study of tidally heated convection. Considering the complexity and the large uncertainties on key properties of icy satellites, we decided to build a generic framework involving a simple modeling of the ice shell. Our modeling, however, includes the most crucial complexities affecting the structure and evolution of ice shells, in particular a strongly temperature-dependent viscosity and mixed (i.e., both basal and internal) heating. Internal heating, here caused by tidal heating, is further assumed to be a function of viscosity. Using this model, we run a series of 70 numerical simulations in 3-D Cartesian geometry, spanning a large range of parameter values. An advantage of this method is that it is not designed for a specific body, so that the obtained numerical results can be easily applied to study a large diversity of problems in various planetary bodies. We first investigate the effects of the different input parameters on the ability to generate melting with particular emphasis on Europa. A limitation of this generic framework is that it does not include the effects of melting on the dynamics (i.e., change of density and viscosity) and the heat budget (i.e., latent heat), while the results indicate a potential to generate a large amount of melt. We therefore run 15 additional 3-D numerical simulations specifically designed to model Europa that account for the effect of melting in the heat budget, following the approach proposed by Choblet et al. (2017). The comparison between the two sets of numerical simulations shows that our simple model, without the effects of melting, is appropriate to study the first occurrence of melting and its location in the ice shell, while the proportion of melting should be assessed using a more complex modeling. We then explore the influence of the presence of impurities in the liquid ocean on our conclusions. Finally, we propose a melting regime diagram of Europa's ice shell and a new assessment of its thermal state. In particular, considering a reasonable value for the amount of tidal heat generated, 1 TW (Hussmann \& Spohn, 2004), combined with a significant extrapolation of our results, we suggest that the ice shell thickness needs to be as thick as $\sim 50-90 \mathrm{~km}$.

\section{Ice Layer as a Tidally Heated Convective System 2.1. Physical Model}

Icy satellites are typically composed of a rocky core, differentiated or undifferentiated (e.g., Sotin \& Tobie, 2004), surrounded by a hydrosphere. The volume of this hydrosphere strongly varies among icy worlds, allowing the presence of different phases of ice, depending on the size of the body. All cases involve ice Ih near the surface, possibly above an ocean of liquid water (Nimmo \& Pappalardo, 2016), while, for the largest objects, denser high-pressure polymorphs of ice can be present beneath the ocean (e.g., Vance et al., 2018). If impurities behaving as antifreeze, such as ammonia or methanol, are initially present in the outer layer, the existence of a contemporary subsurface ocean is very likely (e.g., Deschamps \& Sotin, 2001; Kamata, 2018; Spohn \& Schubert, 2003). Importantly, subsurface oceans have been inferred in many icy satellites, Europa 
(Khurana et al., 1998), Enceladus (Porco et al., 2006), Ganymede (Kivelson et al., 2002), Titan (Lorenz et al., 2008), and Callisto (Khurana et al., 1998), based on different types of data. Following these observations, we consider a system consisting in an outer ice layer above a subsurface ocean.

To model convection within this ice layer, we assume the Boussinesq approximation (Boussinesq, 1903), where the fluid thermodynamical properties are constant, except for density that depends on temperature in the buoyancy force. This assumption is particularly appropriate for ice layers, where relatively small variations of pressure and temperature are involved. We furthermore assume a temperature-dependent viscosity following the classical Frank-Kamenetskii approximation:

$$
\eta^{*}=\eta_{0}^{*} \exp (-E(T-0.5))
$$

with $\eta^{*}$ the dynamic viscosity, $T$ the dimensionless temperature, $\eta_{0}^{*}$ the reference (dynamic) viscosity at the dimensionless reference temperature 0.5 , and $E$ the dimensionless activation energy. With this definition, $E=\ln (\Delta \eta)$, where $\Delta \eta$ is the total viscosity contrast across the ice layer. Note that, here and hereafter, all the properties are denoted with *, while their dimensionless form are denoted without *. The rheology of ice Ih is probably best described by the composite viscosity framework involving four different creep mechanisms (Goldsby \& Kohlstedt, 2001), some of which being highly nonlinear. In addition, grain size is expected to evolve depending on the creep mechanism with potentially strong effects on the ice shell viscosity and its dynamics (e.g., Běhounková et al., 2013). In order to keep simulations tractable and to maintain a more general framework allowing comparison with earlier studies, we adopt a simpler formulation. It is, however, important to keep in mind that considering a different rheology would affect the heat transfer and dynamics of the ice shell (e.g., Harel et al., 2020; Reese et al., 1999). Other effects that may impact the dynamics are ice porosity (filled with void) and ice composition (salt content) near Europa's surface. In the framework of "subduction," Johnson et al. (2017) investigated the combined effects of such features and a temperature-dependent thermal expansion coefficient. They found an important impact on the buoyancy of slabs and thus their dynamics. As this is not a possibility investigated in the present study, and in order to maintain a simple thermodynamical framework (Boussinesq approximation), we do not consider such complexities. For water ice Ih, the viscosity contrast $\Delta \eta$ is estimated to be $10^{7}-10^{8}$ (Durham et al., 2010; Goldsby $\&$ Kohlstedt, 2001). Such high-viscosity contrasts induce very weak convective current for thin ice shells. To overcome this issue, lower-viscosity contrasts from $10^{2}$ to $10^{7}$ are generally assumed (Allu Peddinti \& McNamara, 2015; Barr \& Hammond, 2015; Běhounková et al., 2010). For a viscosity contrast in the range $10^{2}-10^{4}$, sluggish-convection operates and produces a relatively fast resurfacing. While ongoing global-scale surface mobility is reported in the case of Europa (Howell \& Pappalardo, 2018; Kattenhorn \& Prockter, 2014; Mével \& Mercier, 2005), it has been argued that convective processes might not be responsible for this mobility (Howell \& Pappalardo, 2019). Sluggish-lid regime is therefore probably not pertinent for ice shells. Here, we focus on the so-called stagnant lid regime obtained for a viscosity contrast larger than $10^{4}$ (e.g., Davaille \& Jaupart, 1993; Moresi \& Solomatov, 1995; Ogawa et al., 1991). This regime is characterized by the formation of a thick thermally conductive and mechanically rigid lid on top of an almost isoviscous convective interior. The conductive lid accommodates large viscosity and temperature variations, and its thickness is mainly controlled by the amplitude of the viscosity change and by the vigor of convection (Rayleigh number). Based on all these constraints, we restrict our study to viscosity ratios in the range $10^{4}-10^{6}$.

The surface temperature has been determined for different icy satellites. For Europa (Ojakangas \& Stevenson, 1989; Spencer et al., 1999; Trumbo et al., 2018), it is expected to vary spatially by several tens of kelvins. The potential temperature variations should however occur over a large spatial scale, typically hundreds of kilometers, so that locally the surface temperature may be considered as homogeneous. For the sake of simplicity, we therefore consider an ice layer with a constant surface and basal temperature, the former being colder than the latter. The thermal conductivity of ice depends on temperature (e.g., Andersson \& Suga, 1994), which may affect the evolution of ice shells. For simplicity, we did not account for this dependence, but we discuss its possible impact in section 4 . The heat transfer mechanism depends on the ice layer properties, and its vigor can be quantified by the Rayleigh number:

$$
R a_{1 / 2}=\frac{\rho^{*} g^{*} \alpha^{*} \Delta T^{*} d^{* 3}}{\kappa^{*} \eta_{0}^{*}}
$$


where $\rho^{*}$ is the density, $g^{*}$ the acceleration of gravity, $\alpha^{*}$ the thermal expansion coefficient, $\Delta T^{*}$ the temperature contrast across the fluid layer, $d^{*}$ the layer thickness, and $\kappa^{*}$ the thermal diffusivity. Note that in equation (2) the Rayleigh number is defined at viscosity $\eta_{0}^{*}$ corresponding to the median temperature $T_{1 / 2}^{*}=T_{\text {surf }}^{*}+\Delta T^{*} / 2\left(T_{1 / 2}=0.5\right)$. For values of the Rayleigh number lower than a threshold value, heat is transferred by conduction, while it is transferred by thermal convection for larger values. Appropriate values of $R a_{1 / 2}$ are difficult to estimate because of large uncertainties on ice layer properties. Here, we consider different values for the Rayleigh number established using different factors: (i) the potential range of values estimated for different icy satellites, (ii) the necessity to assume a Rayleigh number large enough to have vigorous convection, and (iii) the difficulty to generate melting for thick ice layers.

In addition to basal heating, we assume that the ice layer is tidally heated. Tidal heating produces a heterogeneous heating pattern and its amplitude strongly depends on the orbit of the icy moons, their internal structure, and properties (e.g., Choblet et al., 2017;Kamata et al., 2015;Tobie et al., 2005) such that a detailed modeling of their evolution requires to couple orbital and thermal evolution (e.g., Běhounková et al., 2010; Han \& Showman, 2010, 2011; Hussmann \& Spohn, 2004). Because of this coupling, the evolutions of icy moons depend not only on their own properties but also on the properties of the planet around which they orbit and its other satellites. Alternatively, one may use a collection of precalculated models, each of these models being representative of a short-term orbital evolution of this satellite at a given location of the satellite on its orbit, to constrain the long-term evolution of icy moons. Here, we follow this approach and, as suggested by Tobie et al. (2003), we assume that the tidal heating within the outer ice layer varies with the viscosity following:

$$
H_{\text {tidal }}^{*}=\frac{2 H_{\text {max }}^{*} \eta / \eta_{\text {melt }}}{1+\left(\eta / \eta_{\text {melt }}\right)^{2}},
$$

where $H_{\text {tidal }}^{*}$ is the local heating rate caused by tidal heating, $H_{\max }^{*}$ is the maximum value of the heating rate produced for $\eta=\eta_{\text {melt }}$, with $\eta_{\text {melt }}$ the viscosity close to the melting point, which, in our case, corresponds to the bottom viscosity. Note, however, that the viscosity, or equivalently the temperature, for which the tidal dissipation is maximum can change following the rheology considered (Han \& Showman, 2010). This would change the location where tidal heating is maximum, the pattern of tidal heating, and the total amount of heat generated. For instance, if we assume that the maximum tidal dissipation is reached for a larger temperature than that assumed in equation (3), the quantity of melting would increase, whereas for a lower temperature melting would be generated less easily (Howell \& Pappalardo, 2018; Mitri \& Showman, 2005). Nevertheless, we adopt a modeling where this temperature is fairly low, which may slightly underestimate the generation of melting, in order to obtain more conservative results on the occurrence of melting. Furthermore, this approach, unlike models coupling thermal convection and tidal dissipation (e.g., Běhounková et al., 2010; Han \& Showman, 2010), has the advantage that it does not require to specify a priori the properties of the icy satellite considered.

In this approach, $H_{\max }^{*}$ is an input parameter, while the total amount of tidal heat generated cannot be estimated a priori. This amount is depending on $H_{\text {max }}^{*}$ and on the precise thermal structure of the convective system. It is therefore useful to define a "volume average tidal heating rate" $H_{\text {avg }}^{*}$ corresponding to the homogeneous heating rate required to produce the equivalent amount of heat generated by tidal heating. Note that in Cartesian geometry the conservation of energy implies that

$$
\phi_{\text {top }}^{*}=\phi_{b o t}^{*}+H_{\text {avg }}^{*} d^{*}
$$

where $\phi_{t o p}^{*}$ and $\phi_{b o t}^{*}$ are the surface and bottom heat flux, respectively. This indicates that the surface heat flux is larger than the bottom heat flux, which is, at steady state, a combination of the heat flux from the rocky core and possibly the heat generated within the subsurface ocean. We investigate a large range of values for $H_{\max }^{*}$. The lower bound corresponds to the value required to obtain melting for thin ice shells, the most favorable case for melting, while the upper bound corresponds to the value required to obtain $\phi_{b o t}^{*} \approx 0$. Although potentially interesting, we decided to not consider cases with $\phi_{b o t}^{*}<0$, for which the ice shell is heating the subsurface ocean.

\subsection{Numerical Model}

The numerical simulations are conducted in 3-D Cartesian geometry using the numerical code StagYY (Tackley, 2008). This code solves the dimensionless conservation equations of mass, momentum, and energy for the physical model described in section 2.1. The properties of the system are made dimensionless using 
Table 1

Input Parameters of Numerical Simulations: Rayleigh Number $\left(\mathrm{Ra}_{1 / 2}\right.$ ), Dimensionless Maximum Heating Rate ( $H_{\text {max }}$, See Text for More Details), Viscosity Contrast Across the Ice Layer $(\Delta \eta)$

\begin{tabular}{|c|c|c|c|c|c|c|c|c|c|c|c|c|}
\hline$R a_{1 / 2}$ & $H_{\max }$ & $\Delta \eta$ & $\begin{array}{c}\text { Elapsed } \\
\text { time }\end{array}$ & $R a_{m}$ & $\begin{array}{c}\text { Surface } \\
\text { heat flux }\end{array}$ & $\begin{array}{c}\text { Bottom } \\
\text { heat flux }\end{array}$ & $H_{a v g}$ & $\theta_{m}$ & $\begin{array}{c}d_{w}^{*} \\
(\mathrm{~km})\end{array}$ & $\begin{array}{l}A_{w} \\
(\%)\end{array}$ & $\begin{array}{c}d_{c}^{*} \\
(\mathrm{~km})\end{array}$ & $\begin{array}{l}A_{c} \\
(\%)\end{array}$ \\
\hline 1,500 & 0.7 & $10^{4}$ & 16.33 & $1.91 \times 10^{4}$ & 1.54 & 1.35 & 0.19 & 0.776 & 5.2 & 0.16 & 1 & I \\
\hline 1,500 & 0.9 & $10^{4}$ & 6.797 & $2.29 \times 10^{4}$ & 1.62 & 1.35 & 0.27 & 0.796 & 5.0 & 0.56 & l & I \\
\hline 1,500 & 1.0 & $10^{4}$ & 9.729 & $2.47 \times 10^{4}$ & 1.63 & 1.32 & 0.31 & 0.804 & 3.1 & 0.91 & I & l \\
\hline 1,500 & 1.2 & $10^{4}$ & 5.597 & $2.86 \times 10^{4}$ & 1.67 & 1.28 & 0.39 & 0.820 & 3.0 & 1.83 & I & I \\
\hline 1,500 & 1.25 & $10^{4}$ & 3.767 & $3.05 \times 10^{4}$ & 1.68 & 1.27 & 0.41 & 0.827 & 3.0 & 2.06 & l & I \\
\hline 1,500 & 1.3 & $10^{4}$ & 15.45 & $3.11 \times 10^{4}$ & 1.69 & 1.25 & 0.44 & 0.829 & 2.9 & 2.67 & I & I \\
\hline 1,500 & 1.8 & $10^{4}$ & 11.44 & $4.66 \times 10^{4}$ & 1.80 & 1.10 & 0.70 & 0.873 & 2.8 & 9.36 & 2.9 & 2.68 \\
\hline 1,500 & 2.1 & $10^{4}$ & 5.485 & $6.31 \times 10^{4}$ & 1.87 & 0.97 & 0.90 & 0.906 & 2.7 & 19.9 & 2.8 & 10.0 \\
\hline 1,500 & 2.5 & $10^{4}$ & 12.88 & $8.96 \times 10^{4}$ & 1.95 & 0.75 & 1.20 & 0.944 & 2.6 & 40.2 & 2.7 & 30.8 \\
\hline 1,500 & 3 & $10^{4}$ & 7.640 & $1.29 \times 10^{5}$ & 2.04 & 0.47 & 1.57 & 0.984 & 2.5 & 63.6 & 2.5 & 57.7 \\
\hline 1,500 & 3.5 & $10^{4}$ & 8.846 & $1.69 \times 10^{5}$ & 2.13 & 0.21 & 1.92 & 1.013 & 2.4 & 79.8 & 2.4 & 76.5 \\
\hline 10,000 & 2.0 & $10^{4}$ & 3.788 & $5.65 \times 10^{5}$ & 3.10 & 1.91 & 1.19 & 0.938 & 10.2 & 0.19 & I & I \\
\hline 10,000 & 2.5 & $10^{4}$ & 3.455 & $6.92 \times 10^{5}$ & 3.16 & 1.54 & 1.62 & 0.960 & 8.0 & 1.13 & l & I \\
\hline 10,000 & 2.8 & $10^{4}$ & 3.556 & $7.80 \times 10^{5}$ & 3.23 & 1.34 & 1.89 & 0.973 & 3.8 & 2.55 & I & I \\
\hline 10,000 & 3 & $10^{4}$ & 4.114 & $8.32 \times 10^{5}$ & 3.25 & 1.19 & 2.06 & 0.980 & 3.4 & 5.28 & 10.7 & 0.00 \\
\hline 10,000 & 3.2 & $10^{4}$ & 6.940 & $9.04 \times 10^{5}$ & 3.31 & 1.06 & 2.25 & 0.989 & 3.1 & 34.3 & 3.7 & 0.51 \\
\hline 10,000 & 3.5 & $10^{4}$ & 6.756 & $9.73 \times 10^{5}$ & 3.38 & 0.88 & 2.50 & 0.997 & 3.0 & 73.6 & 3.2 & 28.7 \\
\hline 10,000 & 3.7 & $10^{4}$ & 3.348 & $1.02 \times 10^{6}$ & 3.42 & 0.76 & 2.66 & 1.002 & 2.9 & 82.6 & 3.0 & 62.0 \\
\hline 10,000 & 4 & $10^{4}$ & 2.084 & $1.09 \times 10^{6}$ & 3.53 & 0.62 & 2.91 & 1.009 & 2.8 & 86.2 & 2.9 & 80.8 \\
\hline 10,000 & 4.5 & $10^{4}$ & 1.942 & $1.20 \times 10^{6}$ & 3.63 & 0.32 & 3.31 & 1.020 & 2.7 & 89.4 & 2.8 & 86.7 \\
\hline 10,000 & 5 & $10^{4}$ & 2.485 & $1.36 \times 10^{6}$ & 3.80 & 0.11 & 3.69 & 1.033 & 2.6 & 92.5 & 2.6 & 90.5 \\
\hline 100,000 & 6 & $10^{4}$ & 0.470 & $8.79 \times 10^{6}$ & 6.66 & 1.62 & 5.04 & 0.986 & 14.7 & 0.38 & 1 & I \\
\hline 100,000 & 6.5 & $10^{4}$ & 0.453 & $9.55 \times 10^{6}$ & 6.85 & 1.32 & 5.53 & 0.995 & 3.8 & 2.73 & I & I \\
\hline 100,000 & 7 & $10^{4}$ & 0.406 & $1.03 \times 10^{7}$ & 6.99 & 0.99 & 6.00 & 1.003 & 3.3 & 57.8 & 3.8 & 1.38 \\
\hline 100,000 & 7.5 & $10^{4}$ & 0.438 & $1.10 \times 10^{7}$ & 7.17 & 0.70 & 6.47 & 1.010 & 3.1 & 85.1 & 3.3 & 34.9 \\
\hline 100,000 & 8 & $10^{4}$ & 0.420 & $1.18 \times 10^{7}$ & 7.34 & 0.44 & 6.90 & 1.018 & 3.1 & 89.0 & 3.1 & 84.1 \\
\hline 1,000 & 1.0 & $10^{5}$ & 9.867 & $6.52 \times 10^{4}$ & 1.60 & 1.31 & 0.29 & 0.863 & 6.1 & 0.53 & 1 & I \\
\hline 1,000 & 1.1 & $10^{5}$ & 22.26 & $6.31 \times 10^{4}$ & 1.58 & 1.26 & 0.32 & 0.860 & 5.3 & 0.87 & I & I \\
\hline 1,000 & 1.27 & $10^{5}$ & 7.430 & $7.33 \times 10^{4}$ & 1.62 & 1.23 & 0.39 & 0.873 & 4.2 & 1.49 & / & I \\
\hline 1,000 & 1.3 & $10^{5}$ & 15.79 & $7.85 \times 10^{4}$ & 1.64 & 1.24 & 0.40 & 0.879 & 4.1 & 1.55 & I & I \\
\hline 1,000 & 1.35 & $10^{5}$ & 9.483 & $8.22 \times 10^{4}$ & 1.65 & 1.22 & 0.43 & 0.883 & 4.0 & 1.74 & I & 1 \\
\hline 1,000 & 2 & $10^{5}$ & 6.609 & $1.48 \times 10^{5}$ & 1.75 & 0.96 & 0.79 & 0.934 & 3.8 & 10.5 & 4.1 & 1.07 \\
\hline 1,000 & 2.5 & $10^{5}$ & 12.10 & $1.57 \times 10^{5}$ & 1.83 & 0.70 & 1.13 & 0.967 & 3.7 & 41.4 & 3.8 & 24.6 \\
\hline 1,000 & 3 & $10^{5}$ & 6.649 & $2.95 \times 10^{5}$ & 1.91 & 0.44 & 1.47 & 0.994 & 3.5 & 68.6 & 3.6 & 59.9 \\
\hline 1,000 & 3.5 & $10^{5}$ & 3.643 & $3.72 \times 10^{5}$ & 2.00 & 0.19 & 1.81 & 1.014 & 3.3 & 82.8 & 3.4 & 78.8 \\
\hline 10,000 & 1.5 & $10^{5}$ & 4.226 & $1.45 \times 10^{6}$ & 3.27 & 2.45 & 0.82 & 0.932 & I & I & 1 & I \\
\hline 10,000 & 3 & $10^{5}$ & 6.002 & $2.51 \times 10^{6}$ & 3.47 & 1.39 & 2.08 & 0.980 & 15.2 & 0.46 & I & I \\
\hline 10,000 & 3.2 & $10^{5}$ & 2.029 & $2.66 \times 10^{6}$ & 3.51 & 1.26 & 2.25 & 0.985 & 15.0 & 0.91 & l & I \\
\hline 10,000 & 3.5 & $10^{5}$ & 2.286 & $2.85 \times 10^{6}$ & 3.59 & 1.07 & 2.52 & 0.991 & 5.1 & 2.09 & l & I \\
\hline 10,000 & 3.7 & $10^{5}$ & 1.947 & $2.99 \times 10^{6}$ & 3.63 & 0.95 & 2.68 & 0.995 & 4.4 & 22.4 & I & I \\
\hline 10,000 & 4 & $10^{5}$ & 2.577 & $3.20 \times 10^{6}$ & 3.70 & 0.76 & 2.94 & 1.001 & 4.2 & 77.5 & 4.7 & 2.09 \\
\hline 10,000 & 4.5 & $10^{5}$ & 2.087 & $3.51 \times 10^{6}$ & 3.82 & 0.47 & 3.35 & 1.009 & 3.8 & 86.7 & 4.0 & 76.1 \\
\hline 10,000 & 5 & $10^{5}$ & 2.073 & $3.94 \times 10^{6}$ & 3.96 & 0.22 & 3.74 & 1.019 & 3.8 & 90.2 & 3.8 & 86.7 \\
\hline 100,000 & 6 & $10^{5}$ & 0.481 & $2.54 \times 10^{7}$ & 6.88 & 1.90 & 4.98 & 0.981 & 33.8 & 0.00 & 1 & I \\
\hline
\end{tabular}


Table 1 (Continued)

\begin{tabular}{|c|c|c|c|c|c|c|c|c|c|c|c|c|}
\hline$R a_{1 / 2}$ & $H_{\max }$ & $\Delta \eta$ & $\begin{array}{c}\text { Elapsed } \\
\text { time }\end{array}$ & $R a_{m}$ & $\begin{array}{l}\text { Surface } \\
\text { heat flux }\end{array}$ & $\begin{array}{l}\text { Bottom } \\
\text { heat flux }\end{array}$ & $H_{\text {avg }}$ & $\theta_{m}$ & $\begin{array}{c}d_{w}^{*} \\
(\mathrm{~km})\end{array}$ & $\begin{array}{l}A_{w} \\
(\%)\end{array}$ & $\begin{array}{c}d_{c}^{*} \\
(\mathrm{~km})\end{array}$ & $\begin{array}{l}A_{c} \\
(\%)\end{array}$ \\
\hline 100,000 & 6.5 & $10^{5}$ & 0.664 & $2.72 \times 10^{7}$ & 7.04 & 1.55 & 5.49 & 0.987 & 33.8 & 0.06 & 1 & / \\
\hline 100,000 & 7 & $10^{5}$ & 0.715 & $2.95 \times 10^{7}$ & 7.20 & 1.20 & 6.00 & 0.994 & 32.4 & 0.45 & I & I \\
\hline 100,000 & 7.5 & $10^{5}$ & 0.739 & $3.16 \times 10^{7}$ & 7.38 & 0.89 & 6.49 & 1.000 & 11.2 & 2.30 & I & I \\
\hline 100,000 & 8 & $10^{5}$ & 0.621 & $3.39 \times 10^{7}$ & 7.56 & 0.60 & 6.96 & 1.006 & 4.5 & 17.1 & 22.0 & 0.01 \\
\hline 100,000 & 8.5 & $10^{5}$ & 0.704 & $3.63 \times 10^{7}$ & 7.73 & 0.33 & 7.40 & 1.012 & 4.5 & 81.7 & 6.0 & 1.94 \\
\hline 100,000 & 9.0 & $10^{5}$ & 0.692 & $3.89 \times 10^{7}$ & 7.91 & 0.10 & 7.81 & 1.018 & 4.0 & 88.1 & 4.5 & 37.4 \\
\hline 1,000 & 1.3 & $10^{6}$ & 4.442 & $3.97 \times 10^{5}$ & 1.88 & 1.40 & 0.48 & 0.933 & 10.4 & 0.14 & I & I \\
\hline 1,000 & 1.5 & $10^{6}$ & 4.499 & $4.43 \times 10^{5}$ & 1.90 & 1.31 & 0.59 & 0.941 & 10.0 & 0.42 & I & I \\
\hline 1,000 & 1.8 & $10^{6}$ & 4.234 & $5.37 \times 10^{5}$ & 1.93 & 1.15 & 0.78 & 0.955 & 8.8 & 1.30 & I & I \\
\hline 1,000 & 2 & $10^{6}$ & 4.448 & $6.00 \times 10^{5}$ & 1.94 & 1.04 & 0.90 & 0.963 & 5.5 & 2.48 & I & I \\
\hline 1,000 & 2.5 & $10^{6}$ & 10.35 & $8.02 \times 10^{5}$ & 2.01 & 0.75 & 1.26 & 0.984 & 5.2 & 32.1 & 10.0 & 0.01 \\
\hline 1,000 & 3 & $10^{6}$ & 4.003 & $9.86 \times 10^{5}$ & 2.08 & 0.48 & 1.60 & 0.999 & 4.8 & 74.7 & 5.0 & 51.1 \\
\hline 1,000 & 3.5 & $10^{6}$ & 3.804 & $1.18 \times 10^{6}$ & 2.18 & 0.24 & 1.94 & 1.012 & 4.5 & 86.2 & 4.6 & 81.1 \\
\hline 1,000 & 4 & $10^{6}$ & 6.513 & $1.37 \times 10^{6}$ & 2.29 & 0.02 & 2.27 & 1.023 & 4.3 & 90.9 & 4.4 & 87.9 \\
\hline 10,000 & 3 & $10^{6}$ & 1.852 & $7.08 \times 10^{6}$ & 3.84 & 1.75 & 2.09 & 0.975 & I & I & 1 & I \\
\hline 10,000 & 3.5 & $10^{6}$ & 2.011 & $8.02 \times 10^{6}$ & 3.95 & 1.41 & 2.54 & 0.984 & 23.0 & 0.02 & l & I \\
\hline 10,000 & 4 & $10^{6}$ & 1.633 & $8.95 \times 10^{6}$ & 4.03 & 1.05 & 2.98 & 0.992 & 21.4 & 0.55 & I & / \\
\hline 10,000 & 4.5 & $10^{6}$ & 0.965 & $1.00 \times 10^{7}$ & 4.14 & 0.72 & 3.42 & 1.000 & 5.7 & 15.2 & 22.8 & 0.00 \\
\hline 10,000 & 4.7 & $10^{6}$ & 1.004 & $1.04 \times 10^{7}$ & 4.20 & 0.60 & 3.60 & 1.003 & 5.6 & 58.6 & 11.7 & 0.03 \\
\hline 10,000 & 5 & $10^{6}$ & 0.960 & $1.10 \times 10^{7}$ & 4.27 & 0.42 & 3.85 & 1.007 & 5.3 & 83.6 & 5.8 & 5.02 \\
\hline 10,000 & 5.5 & $10^{6}$ & 1.033 & $1.23 \times 10^{7}$ & 4.43 & 0.18 & 4.25 & 1.015 & 4.9 & 89.2 & 5.2 & 80.6 \\
\hline 100,000 & 8.0 & $10^{6}$ & 0.355 & $9.22 \times 10^{7}$ & 8.09 & 1.12 & 6.97 & 0.994 & 49.7 & 0.09 & / & / \\
\hline 100,000 & 8.5 & $10^{6}$ & 0.579 & $1.00 \times 10^{8}$ & 8.28 & 0.81 & 7.47 & 1.000 & 48.9 & 1.04 & I & I \\
\hline 100,000 & 9.0 & $10^{6}$ & 0.305 & $1.06 \times 10^{8}$ & 8.45 & 0.51 & 7.94 & 1.004 & 31.3 & 6.66 & I & I \\
\hline 100,000 & 9.5 & $10^{6}$ & 0.500 & $1.13 \times 10^{8}$ & 8.63 & 0.23 & 8.40 & 1.009 & 16.6 & 30.9 & I & I \\
\hline 100,000 & 10.0 & $10^{6}$ & 0.500 & $1.22 \times 10^{8}$ & 8.81 & 0.01 & 8.80 & 1.014 & 7.2 & 82.3 & 33.6 & 0.11 \\
\hline
\end{tabular}

Note. We also report some key characteristics of the numerical simulations: dimensionless time calculated for the numerical simulation, effective Rayleigh number $\left(R a_{m}\right)$, dimensionless top and bottom heat flux, dimensionless average heating rate $\left(H_{\text {avg }}\right)$, dimensionless temperature of the well-mixed interior $\left(\theta_{m}\right)$, minimum depth at which the temperature is exceeding the melting temperature $\left(d_{w}^{*}\right.$ and $\left.d_{c}^{*}\right)$ together with the maximum proportion of material above the melting temperature at a given depth $\left(A_{w}\right.$ and $\left.A_{c}\right)$, calculated for Europa assuming an ocean composed either of pure water (subscript $w$ ) or of a mixture of water and $10 \mathrm{wt} \%$ of $\mathrm{MgSO}_{4}$ (subscript $c$ ), respectively. The symbol "/” indicates that no melting is generated.

the classical characteristic quantities (see Tackley, 2008 for more details). In particular, heating rates are made dimensionless using

$$
H=\frac{H^{*} d^{* 2}}{\lambda^{*} \Delta T^{*}},
$$

where $\lambda^{*}$ is the thermal conductivity, here assumed constant. The top and bottom mechanical boundary conditions of the system are free slip, while the sides are reflecting. The initial temperature condition consists in linear variations of temperature close to the top and bottom surfaces and a constant temperature interior, to which random, small-amplitude, temperature perturbations are superimposed. We stop the numerical simulations when the top and bottom heat fluxes reach a statistical steady state, which is characterized here by temporal fluctuations equal to zero on average. The grid resolution and domain aspect ratio are either $256 \times 256 \times 64$ and $8: 8$ or $512 \times 512 \times 64$ and $4: 4$ or $2: 2$, respectively. With this setup, we conducted 70 simulations varying $R a_{1 / 2}$ in the range $10^{3}-10^{5}, \Delta \eta$ between $10^{4}$ and $10^{6}$, and $H_{\text {max }}$ between 0.7 and 10 (Table 1). A general description of the numerical results is provided in the supporting information. The following sections are dedicated to the application of these results to the hydrosphere of Europa. 


\begin{tabular}{|c|c|c|c|}
\hline Parameter & Symbol & Value & Unit \\
\hline Radius & $R^{*}$ & 1,560 & $\mathrm{~km}$ \\
\hline Rocky core radius & $R_{c}^{*}$ & $\sim 1,400^{\mathrm{a}}$ & $\mathrm{km}$ \\
\hline Gravity acceleration & $g^{*}$ & 1.3 & $\mathrm{~m} / \mathrm{s}^{2}$ \\
\hline Surface temperature & $T_{\text {surf }}^{*}$ & 95 & $\mathrm{~K}$ \\
\hline Expansion coefficient & $\alpha^{*}$ & $1.62 \times 10^{-4 a}$ & $\mathrm{~K}^{-1}$ \\
\hline Density & $\rho^{*}$ & $920^{\mathrm{b}}$ & $\mathrm{kg} / \mathrm{m}^{3}$ \\
\hline Thermal diffusivity & $\kappa^{*}$ & $1.36 \times 10^{-6 c}$ & $\mathrm{~m}^{2} / \mathrm{s}$ \\
\hline Thermal conductivity & $\lambda^{*}$ & $2.5^{\mathrm{c}}$ & $\mathrm{W} \cdot \mathrm{m}^{-1} \cdot \mathrm{K}^{-1}$ \\
\hline Latent heat of fusion & $L^{*}$ & $284 \times 10^{3 \mathrm{~d}}$ & $\mathrm{~J} / \mathrm{kg}$ \\
\hline Viscosity close to melting point & $\eta_{\text {melt }}^{*}$ & $3 \times 10^{13 \mathrm{e}}$ & $\mathrm{Pas}$ \\
\hline
\end{tabular}

\section{Melting in Icy Satellites}

The simulations listed in Table 1 may, in principle, be applied to all icy bodies. It is, however, interesting to illustrate them with a specific example. Furthermore, these simulations do not account for the effects of melt itself on the heat budget. To assess the occurrence and distribution of melt within the ice shell of icy bodies, we therefore proceed in two steps. First, we apply our results to Europa, within which presence of reservoirs of partially molten material close to the surface has been suggested (e.g., Dombard et al., 2013; Manga \& Michaut, 2017; Michaut \& Manga, 2014) and whose physical properties are relatively well constrained. Note that two scheduled missions, Europa Clipper (National Aeronautics and Space Administration) and JUICE (European Space Agency), may bring additional constraints refining Europa's internal structure. Second, we run another set of numerical simulations in which the effect of melting in the heat budget is modeled following the approach proposed by Choblet et al. (2017). In contrast to the calculations listed in Table 1, these additional simulations are bound to Europa and cannot be used to study other bodies.

Estimating the amount and location of melting as a function of input parameters requires to identify, in each numerical simulation, the regions where the local temperature is higher than the melting temperature. A first step is therefore to dimensionalize the temperature fields and spatial dimensions of our numerical simulations. The scale used for the spatial dimensions is the ice layer thickness $\left(d^{*}\right)$, a property difficult to estimate and whose value is very uncertain in most icy satellites (e.g., Anderson et al., 1997, 1998; Kuskov \& Kronrod, 2005). In addition, the average ice layer thickness is expected to evolve throughout the moon's history (e.g., Allu Peddinti \& McNamara, 2019; Hussmann \& Spohn, 2004). At a given time, the ice layer may further vary laterally. For objects such as Enceladus, and owing to a small internal pressure, lateral variations in ice shell thickness could be as large as the average value (e.g., Čadek et al., 2019), provided that the viscosity is sufficiently high to overcome the viscous relaxation. In the specific case of Europa, numerical models typically report lateral variations in the ice shell thickness in the range 5-10 km (Tobie et al., 2003), while more recent studies suggest that preserving such variations against viscous relaxation would require unrealistically large viscosities (Čadek et al., 2017). Due to these uncertainties, we investigate a relatively large range of values dictated by the choice of dimensionless parameters introduced above. According to equation (2), the ice layer thickness can be expressed as a function of the input parameters of our models following

$$
d^{*}=\left(\frac{R a_{1 / 2} \kappa^{*} \eta_{0}^{*}}{\rho^{*} g^{*} \alpha^{*} \Delta T^{*}}\right)^{1 / 3}
$$

where $\eta_{0}^{*}=\eta_{\text {melt }}^{*} \Delta \eta^{1 / 2}$, with $\eta_{\text {melt }}^{*}$ being the viscosity of ice close to its melting point and $\Delta \eta=\exp (E)$. Note that by definition, $\eta_{\text {melt }}^{*}$ is also the viscosity at the bottom of the ice layer. Assuming an ice Ih composition for the ice shell, parameters of equation (6) are well constrained (Table 2), except for $\eta_{\text {melt }}^{*}$ and the temperature 

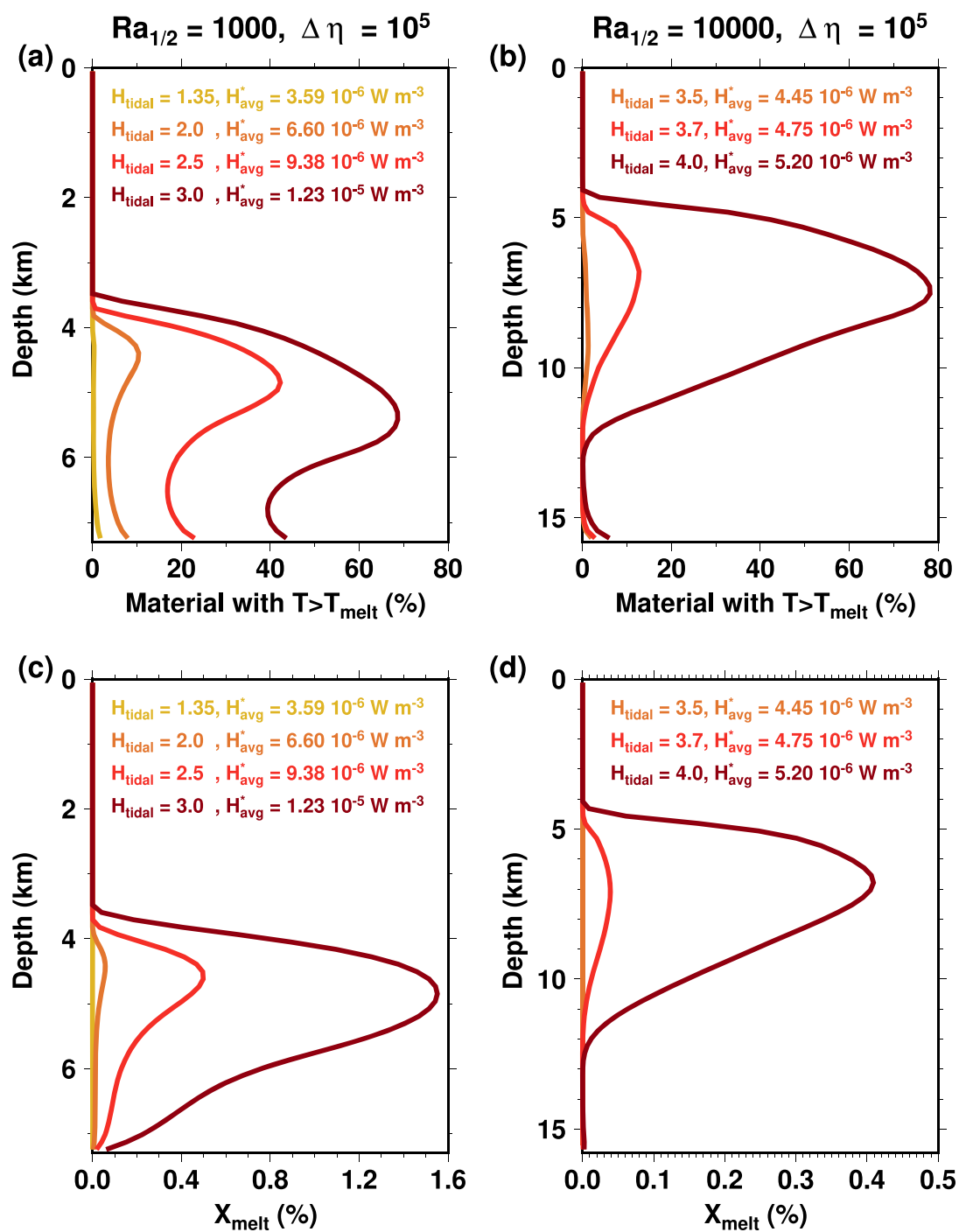

Figure 1. (a, b) Proportion of material above the melting temperature and (c, d) average potential melting rate (see text for more details) as a function of depth for two different values of the Rayleigh number. For both cases, we show the results obtained with different values of the tidal heating rate. In (a) and (b), a proportion of 100\% and 0\% means the material is fully above the melting temperature or fully solid, respectively. Calculations are done using the properties of Europa given in Table 2, and the thickness of the ice layer is constrained from the assumed value of $R a_{1 / 2}$. Here, the subsurface ocean is composed of pure water, and the melting temperature is given by equation (7).

at the base of the ice layer $T_{b o t}^{*}$. The viscosity close to the melting point has been estimated by previous studies based either on the observations of flow features (e.g., Jankowski \& Squyres, 1988; Melosh \& Janes, 1989; Nimmo \& Manga, 2002; Showman \& Han, 2004) or on deformation experiments (e.g., Arakawa \& Maeno, 1994; Goldsby \& Kohlstedt, 2001) and it has been suggested to be in the range $10^{12}-10^{15} \mathrm{~Pa}$ s. Here, we assume that $\eta_{\text {melt }}^{*}=310^{13} \mathrm{~Pa} \mathrm{~s}$, but it is important to keep in mind that large uncertainties remain on its exact value. The temperature contrast across the ice layer, which is also the temperature scale necessary to dimensionalize the temperature fields, can be calculated from the surface temperature $\left(T_{\text {surf }}^{*}\right.$, listed in Table 2) and the temperature at the bottom of the ice layer $\left(T_{b o t}^{*}\right)$. Dimensionalizing the numerical results therefore depends on the determination of $T_{b o t}^{*}$. Assuming the presence of a subsurface ocean, this bottom temperature is given by the melting temperature at that depth.

\subsection{Occurrence of Melting}

We first assume that the ice layer and the subsurface ocean are both composed of pure water (effects of impurities will be investigated in section 3.3). Following Chizhov (1993), the melting temperature of pure 
water, $T_{\text {melt }}^{*}$, can be expressed as

$$
T_{\text {melt }}^{*}=273.2\left(1-\frac{p^{*}}{395}\right)^{1 / 9},
$$

where $p^{*}$ is pressure in megapascals. To calculate pressure at a given depth $\left(d_{i}^{*}\right)$, we assume that density is constant with pressure and that pressure is given by $p^{*}=\rho^{*} g^{*} d_{i}^{*}$. Equation (7) gives $T_{b o t}^{*}$, the temperature at the interface between the ice layer and the subsurface ocean, since it corresponds, by definition, to the melting temperature at that depth. This, in turn, gives access to the temperature contrast across the ice layer.

In order to identify regions where melting is present, the dimensional temperature fields are compared to the melting temperature. In Figures $1 \mathrm{a}$ and $1 \mathrm{~b}$, we plotted the average amount of material above the melting temperature as a function of depth for different values of the tidal heating rate and for two different values of $\left(R a_{1 / 2}, \Delta \eta\right)$. For information, we also report in Table 1 the minimum depth where melting may be generated together with the maximum proportion of material above the melting temperature for each numerical simulation. Interestingly, the results reported in Table 1 and Figures 1a and $1 \mathrm{~b}$ show that the volume fraction of the temperature field above the melting point increases very sharply with increasing tidal heating rates. For instance, in Figure 1b, the maximum amount of material above the melting temperature at a given depth is about $15 \%$ and $84 \%$, for $H_{\text {avg }}$ of $4.75 \times 10^{-6}$ and $5.20 \times 10^{-6} \mathrm{~W} / \mathrm{m}^{3}$, respectively. This effect is, however, less pronounced for very thin ice layers, as shown in Figure 1a, where the amount of tidal heat generated would need to double to observe a similar effect. It is, however, important to keep in mind that the effect of latent heat is not considered in these models, so that the amount of material above the melting point is not indicative of actual melting. One way to estimate the potential amount of melting is by calculating the amount of heat available for melting. To achieve this, one identifies every parcel of fluid with a temperature $T^{*}$ above the local melting temperature $T_{\text {melt }}^{*}$ and calculate the excess of heat. Assuming that all the excess of heat is used to produce melting, we obtain the local production of melt:

$$
\delta X_{\text {melt }}^{*}=\frac{C_{p}^{*}}{L^{*}}\left(T^{*}-T_{m e l t}^{*}\right),
$$

with $C_{p}^{*}=\lambda^{*} /\left(\rho^{*} \kappa^{*}\right)$ the specific heat capacity and $L^{*}$ the latent heat of fusion. This potential production of melt is then laterally averaged and reported in Figures $1 \mathrm{c}$ and $1 \mathrm{~d}$. One interesting outcome of this figure is the low $X_{\text {melt }}^{*}$ predicted at the base of the layer. In this area, although there is a large proportion of material with a temperature larger than the melting temperature, the temperature excess involved is generally low so that only a low proportion of melting is expected. Because the effect of the latent heat is only accounted for a posteriori, it is difficult to assess the relevance of the potential production of melt calculated here. Furthermore, the simplicity of our numerical model implies that results with a large amount of material above the melting temperature may not be applicable. In the remainder of this section, we therefore do not attempt to constrain the actual amount of melting but rather focus on the first occurrence of melting, that is, values of $H_{\max }$ large enough to produce melting but low enough to avoid the complete melting of part of the ice layer.

A second important result shown in Figure 1 is that the two cases are characterized by a different temperature distribution. In Figure 1b, temperatures exceeding the melting point appears in the upper half of the layer, as illustrated in Figure 2b, while in Figure 1a, such hot regions appear at the base of the layer and then possibly propagates upward to form wide plumes (Figure 2a). However, the occurrence of melting would induce the formation of negatively buoyant parcels of liquid water, an effect not modeled here but that may significantly affect the dynamics of upwelling warm ice instabilities (Tobie et al., 2003). As a result, we expect that the location of temperature values above the melting point indicated by our results, especially the minimum depth, may not be relevant when the melt distribution is similar to that in Figure 1a. By contrast, for temperature distributions as those displayed in Figure $1 \mathrm{~b}$ the minimum depth, where melting could be generated, and the depth at which maximal melting occur are robust features, even though accounting for the effect of melt on the bulk ice matrix density could add oscillatory phenomena (Tobie et al., 2003). As a consequence, we report in Figure 3 the results obtained for all the conditions explored, but we will only describe the cases similar to Figure $2 b$, where melt pockets are generated.

Interestingly, and independently of the viscosity contrast, melt pockets are generated only when the ice layer is moderately thick ( $15 \leq d^{*} \leq 35 \mathrm{~km}$, Figures $3 \mathrm{c}, 3 \mathrm{e}, 3 \mathrm{f}$, and $\left.3 \mathrm{~h}\right)$. For these conditions, melt pockets are 
(a)

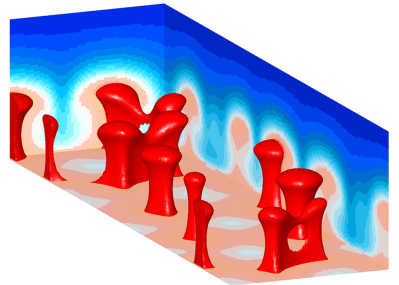

(b)

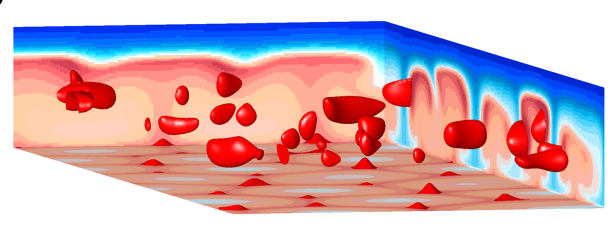

Figure 2. Portions of the temperature field where the isosurfaces highlight the material above the melting temperature. Background colors denote the temperature distribution on the box boundaries, where red and blue correspond to hot and cold material, respectively, and a logarithmic color scale is applied to enhance the visibility of the convective interior. The results are computed for Europa using numerical simulations conducted with (a) $\Delta \eta=10^{5}, R a_{1 / 2}=1,000$ and $H_{\max }=2.0$ (corresponding to a thickness of $\sim 7 \mathrm{~km}$ ) and (b) $\Delta \eta=10^{5}, R a_{1 / 2}=10,000$ and $H_{\max }=3.5$ (corresponding to a thickness of $\sim 16 \mathrm{~km}$ ). The model assumes that the subsurface ocean is composed of pure water, and the melting temperature of water is given by equation (7).

transient features located right below the conductive lid. Therefore, following the change of the conductive lid thickness, the minimum depth where melt is generated slightly increases with the viscosity contrast and the ice layer thickness while remaining fairly shallow (around $4-6 \mathrm{~km}$ ). The fate of these putative melt pockets is, however, difficult to determine. For such shallow depths, it is possible that part of the melt reaches the surface (Lesage et al., 2020; Mitri et al., 2008; Showman \& Han, 2004), while most of the melt probably sinks to the subsurface ocean via percolation through the solid ice matrix (Kalousová et al., 2014, 2016). The occurrence of these melt pockets may therefore favor material exchange between the subsurface ocean, the ice layer, and potentially the surface.

\subsection{Effects of Melting on the Heat Transfer Within the Ice Shell}

The previous results have been established using an approach that estimates the occurrence of melting a posteriori, implying that the effects of melting on the heat budget of the ice shell were neglected. A more appropriate description of melting might significantly alter our conclusions. To assess the robustness of our results, we have conducted an additional set of numerical simulations including the effects of melting on the heat budget.

For this, we follow the description of the melting effects proposed by Choblet et al. (2017). In this approach, it is assumed that the melt is instantaneously extracted from the ice shell, which is valid when melt transport occurs in a much faster timescale than the convective motion, in line with the results of Kalousová et al. (2014) obtained with a full description of the two-phase flow. In that case, all the material above the local melting temperature $\left(T_{\text {melt }}^{*}\right)$ is set back at $T_{\text {melt }}^{*}$. This process is equivalent to remove heat from the system, and the associated heat loss $\left(P_{\text {melt }}^{*}\right)$ represents the amount of energy available to produce melt, which can be calculated at every time step using

$$
P_{\text {melt }}^{*}=\int_{V^{*}} \rho_{l i q}^{*} C_{p}^{*}\left(T^{*}-T_{m e l t}^{*}\right) \delta t^{*-1} \mathrm{~d} V^{*}
$$

where $V^{*}$ is the volume of the ice shell, $\rho_{\text {liq }}^{*}=1,000 \mathrm{~kg} / \mathrm{m}^{3}$ the density of liquid water, $C_{p}^{*}=\lambda^{*} /\left(\rho^{*} \kappa^{*}\right)$ the specific heat capacity, $T^{*}$ the value of the local temperature when above the melting temperature, and $\delta t^{*}$ the time difference between the time steps. The proportion of melt $\left(a_{\text {melt }}^{*}\right)$ can subsequently be estimated using

$$
a_{m e l t}^{*}=\frac{P_{m e l t}^{*}}{\rho_{\text {liq }}^{*} L^{*} V^{*}},
$$

where $L^{*}$ is the latent heat of fusion and $V^{*}$ the volume of the ice shell considered. This relationship provides the proportion of the ice shell melting per second. For convenience, $a_{\text {melt }}^{*}$ may be converted in $\% / \mathrm{Myr}$ indicating the proportion (in percent) of the ice shell melting every million years.

While in section 3.1 the melting profile has been considered a posteriori to infer the possible occurrence of melting, in this new framework, where the melting effect is accounted for in the conservation of energy, the melting temperature has to be prescribed as an input parameter. Note that, since the melting profile depends on parameters such as the thickness of the ice shell and the ocean composition, the simulations presented here are not as generic as the subsolidus convection runs mentioned above. For the sake of exam- 


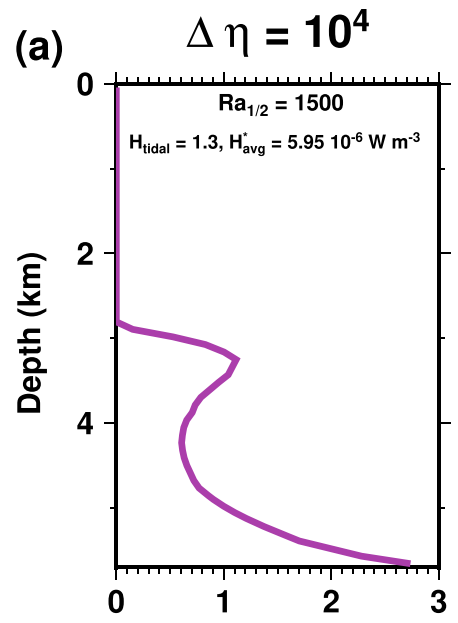

(b) Material with $\mathrm{T}>\mathrm{T}_{\text {melt }}(\%)$

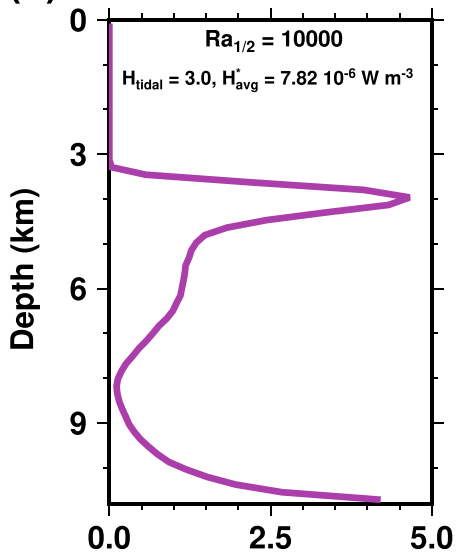

(c) Material with $\mathrm{T}>\mathrm{T}_{\text {melt }}(\%)$

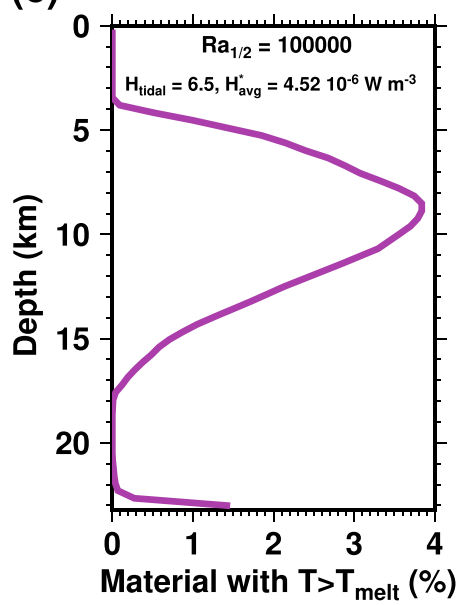

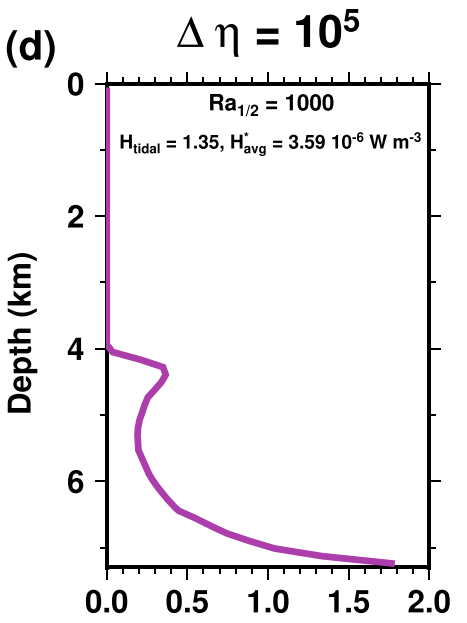
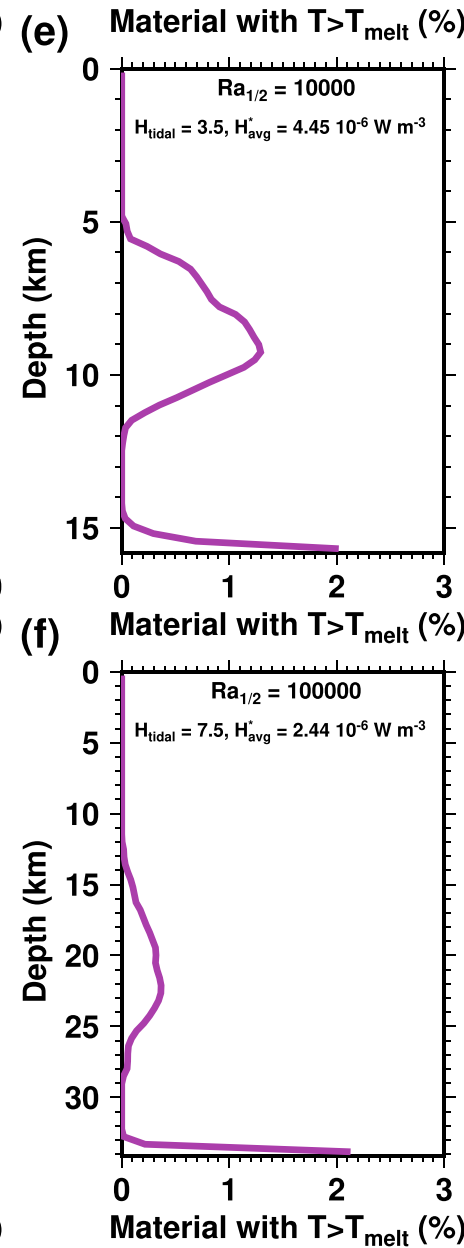

(h)
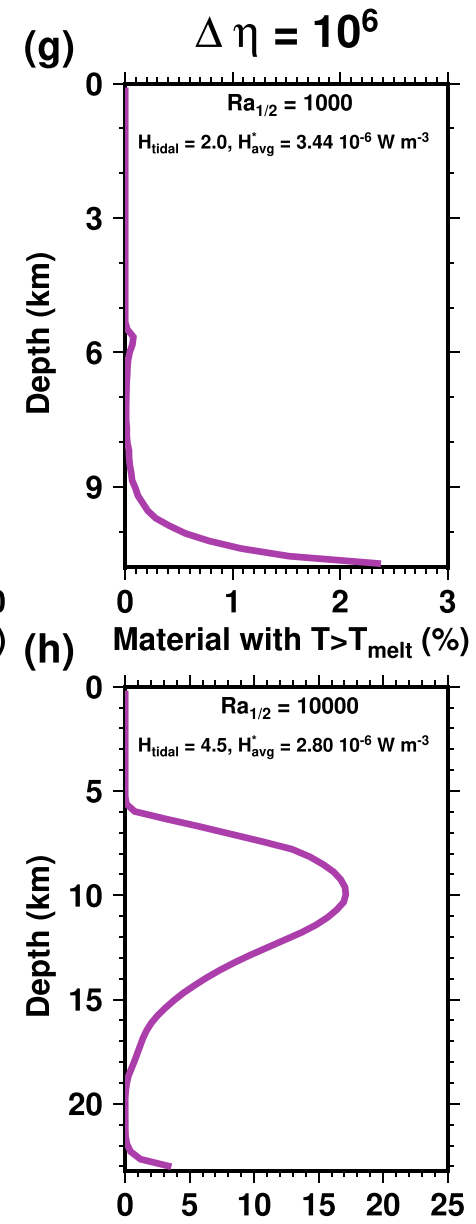

(h) Material with $\mathrm{T}>\mathrm{T}_{\text {melt }}(\%)$
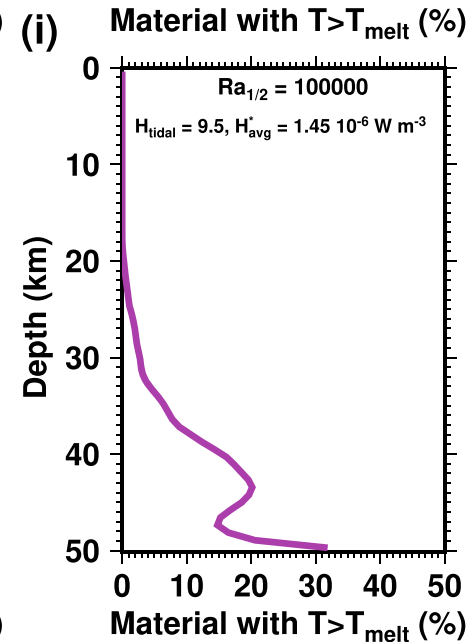

Figure 3. (a-i) Proportion of material above the melting temperature as a function of depth for different cases. A proportion of $100 \%$ and $0 \%$ means the material is fully above the melting temperature or fully solid, respectively. Calculations are done using the properties of Europa given in Table 2, and the thickness of the ice layer is constrained from the assumed value of $R a_{1 / 2}$. Here, the subsurface ocean is composed of pure water, and the melting temperature is given by equation (7). 
Table 3

Numerical Simulations Including the Effects of Melting Following Choblet et al. (2017)

\begin{tabular}{|c|c|c|c|c|c|c|c|c|c|c|c|c|c|}
\hline \multirow[t]{2}{*}{$R a_{1 / 2}$} & \multirow[t]{2}{*}{$\begin{array}{c}H_{\max }^{*} \\
\left(\mathrm{~W} / \mathrm{m}^{3}\right)\end{array}$} & \multirow[t]{2}{*}{$\begin{array}{c}\mathrm{d}^{*} \\
(\mathrm{~km})\end{array}$} & \multirow[t]{2}{*}{$\begin{array}{l}\mathrm{T}_{b o t}^{*} \\
(\mathrm{~K})\end{array}$} & \multicolumn{2}{|c|}{$\begin{array}{l}\theta_{m}^{*} \\
(\mathrm{~K})\end{array}$} & \multicolumn{2}{|c|}{$\begin{array}{c}\phi_{t o p}^{*} \\
\left(\mathrm{~mW} / \mathrm{m}^{2}\right)\end{array}$} & \multicolumn{2}{|c|}{$\begin{array}{c}\phi_{b o t}^{*} \\
\left(\mathrm{~mW} / \mathrm{m}^{2}\right)\end{array}$} & \multicolumn{2}{|c|}{$\begin{array}{c}H_{\text {avg }}^{*} \\
\left(10^{-6} \mathrm{~W} / \mathrm{m}^{3}\right)\end{array}$} & \multirow{2}{*}{$\begin{array}{c}P_{\text {melt }}^{*} \\
(\mathrm{TW}) \\
(2)\end{array}$} & \multirow{2}{*}{$\begin{array}{c}a_{m e l t}^{*} / \mathrm{d}^{*} \\
(\% / \mathrm{Myr}) \\
(2)\end{array}$} \\
\hline & & & & $(1)$ & (2) & $(1)$ & $(2)$ & (1) & (2) & $(1)$ & (2) & & \\
\hline 1,000 & $9.16 \times 10^{-6}$ & 7.3 & 273 & 248 & 1 & 96 & 1 & 77 & 1 & 2.64 & 1 & 1 & 1 \\
\hline 1,000 & $1.06 \times 10^{-5}$ & 7.3 & 273 & 250 & I & 99 & I & 75 & I & 3.26 & l & I & l \\
\hline 1,000 & $1.08 \times 10^{-5}$ & 7.3 & 273 & 251 & I & 100 & I & 75 & I & 3.40 & I & I & l \\
\hline 1,000 & $1.12 \times 10^{-5}$ & 7.3 & 273 & 252 & 252 & 100 & 101 & 74 & 74 & 3.59 & 3.61 & 0.00 & 0.07 \\
\hline 1,000 & $1.67 \times 10^{-5}$ & 7.3 & 273 & 261 & 260 & 107 & 106 & 58 & 59 & 6.60 & 6.48 & 0.04 & 1.82 \\
\hline 1,000 & $2.08 \times 10^{-5}$ & 7.3 & 273 & 267 & 265 & 111 & 105 & 43 & 47 & 9.38 & 8.76 & 0.18 & 9.03 \\
\hline 1,000 & $2.50 \times 10^{-5}$ & 7.3 & 273 & 272 & 267 & 116 & 105 & 27 & 38 & 12.3 & 10.9 & 0.38 & 18.7 \\
\hline 1,000 & $2.91 \times 10^{-5}$ & 7.3 & 273 & 275 & 268 & 122 & 105 & 12 & 32 & 15.1 & 12.9 & 0.63 & 31.5 \\
\hline 10,000 & $2.65 \times 10^{-6}$ & 15.8 & 272 & 260 & I & 92 & I & 69 & I & 1.45 & / & I & I \\
\hline 10,000 & $5.31 \times 10^{-6}$ & 15.8 & 272 & 268 & I & 97 & I & 39 & I & 3.69 & l & I & l \\
\hline 10,000 & $5.66 \times 10^{-6}$ & 15.8 & 272 & 269 & I & 98 & I & 35 & I & 3.99 & I & I & I \\
\hline 10,000 & $6.19 \times 10^{-6}$ & 15.8 & 272 & 270 & 270 & 100 & 100 & 30 & 30 & 4.45 & 4.45 & 0.00 & 0.08 \\
\hline 10,000 & $6.55 \times 10^{-6}$ & 15.8 & 272 & 271 & 271 & 102 & 101 & 26 & 29 & 4.75 & 4.74 & 0.06 & 1.48 \\
\hline 10,000 & $7.08 \times 10^{-6}$ & 15.8 & 272 & 272 & 271 & 103 & 102 & 21 & 28 & 5.20 & 5.16 & 0.22 & 5.01 \\
\hline 10,000 & $7.96 \times 10^{-6}$ & 15.8 & 272 & 273 & 271 & 107 & 103 & 13 & 28 & 5.93 & 5.82 & 0.54 & 12.3 \\
\hline 10,000 & $8.85 \times 10^{-6}$ & 15.8 & 272 & 275 & 271 & 111 & 102 & 6.1 & 26 & 6.62 & 6.47 & 0.79 & 18.1 \\
\hline 100,000 & $2.26 \times 10^{-6}$ & 34.1 & 270 & 267 & I & 88 & / & 24 & I & 1.87 & / & I & I \\
\hline 100,000 & $2.44 \times 10^{-6}$ & 34.1 & 270 & 268 & I & 90 & I & 20 & I & 2.06 & 1 & / & I \\
\hline 100,000 & $2.63 \times 10^{-6}$ & 34.1 & 270 & 269 & 269 & 92 & 92 & 16 & 15 & 2.25 & 2.25 & 0.00 & 0.00 \\
\hline 100,000 & $2.82 \times 10^{-6}$ & 34.1 & 270 & 270 & 270 & 95 & 95 & 11 & 11 & 2.44 & 2.44 & 0.00 & 0.02 \\
\hline 100,000 & $3.01 \times 10^{-6}$ & 34.1 & 270 & 271 & 271 & 97 & 96 & 7.7 & 9.0 & 2.62 & 2.61 & 0.06 & 0.63 \\
\hline 100,000 & $3.20 \times 10^{-6}$ & 34.1 & 270 & 272 & 271 & 99 & 97 & 4.2 & 8.5 & 2.78 & 2.78 & 0.20 & 2.11 \\
\hline 100,000 & $3.38 \times 10^{-6}$ & 34.1 & 270 & 273 & 271 & 101 & 97 & 1.2 & 8.1 & 2.94 & 2.94 & 0.36 & 3.82 \\
\hline
\end{tabular}

Note. (1) indicates the initial numerical simulations reported in Table 1, while (2) indicates the numerical simulations including melting. We report here values for the Rayleigh number $\left(R a_{1 / 2}\right)$, the maximum heating rate $\left(H_{\max }^{*}\right)$, the thickness of the ice layer $\left(d^{*}\right)$, the temperature at the interface between the ice layer and the subsurface ocean $\left(\mathrm{T}_{b o t}^{*}\right)$, the temperature of the well-mixed interior $\left(\theta_{m}^{*}\right)$, the surface heat flux $\left(\phi_{t o p}^{*}\right)$, the bottom heat flux $\left(\phi_{b o t}^{*}\right)$, the average tidal heating rate $\left(H_{\text {avg }}^{*}\right)$, the amount of heat available for producing melt in the whole ice shell assuming that $d^{*}$ is constant $\left(P_{\text {melt }}^{*}\right)$, and the proportion of the ice layer that should melt per million year $\left(a_{m e l t}^{*}\right)$. The symbol "/” indicates that, because the low amount of melting, the numerical simulation including the effect of melting has not been conducted.

ple, we choose to consider the ice shell of Europa in the pure water case with a viscosity contrast $\Delta \eta=10^{5}$. An exhaustive study would request to investigate different bodies and/or different values of the viscosity contrast. This is, however, out of the scope of this paper, but note that, while we only study these specific conditions, our results can easily be adapted to other cases. We have modified StagYY (Tackley, 2008) following this formalism (see for more details Choblet et al., 2017) and successfully compared our results to those obtained by OEDIPUS (Choblet, 2005; Choblet et al., 2017). Next, we selected the numerical simulations exhibiting a large volume fraction of the temperature field above the melting point (Table 3), and, for each of these simulations, we used their final state as an initial condition for the modified version of StagYY. We ran the numerical simulations until an equilibrium state is reached. Results are reported in Table 3 and Figure 4, along with the corresponding results without melting effects listed in Table 1.

We first observe that, for $\mathrm{d}^{*}=15.8 \mathrm{~km}$ and $d^{*}=34.1 \mathrm{~km}$ (Figures $4 \mathrm{a}$ and $4 \mathrm{~b}$ ), the surface and bottom heat fluxes remain constant for a tidal heating rate above a certain value, corresponding to the threshold value for the generation of a nonnegligible amount of melt. This means that, for a given Rayleigh number and viscosity contrast, tidal heating is used to heat the system up to a critical threshold. Above this threshold, the exceeding tidal heat is only used to produce melt that either percolates back to the ocean or (for a smaller fraction close to the upper boundary if the stress field allows it) reaches the surface. In the case $d^{*}=7.3 \mathrm{~km}$, nevertheless, the bottom heat flux keeps decreasing with increasing tidal heating rate (Figure 4d), while the surface 
(a)

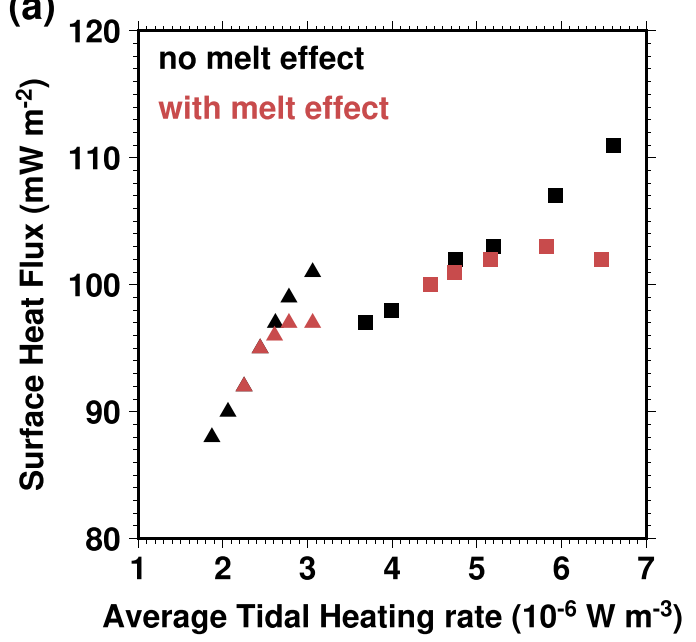

(b)

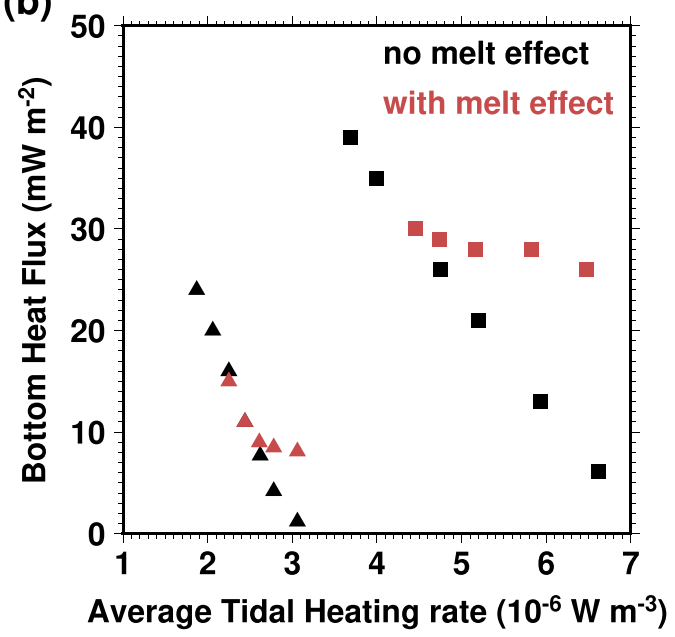

(c)

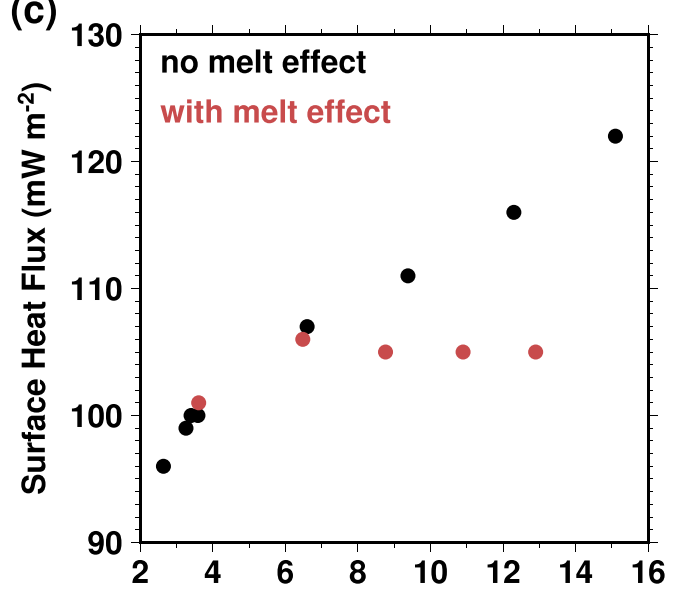

Average Tidal Heating rate $\left(10^{-6} \mathrm{~W} \mathrm{~m}^{-3}\right)$ (d)

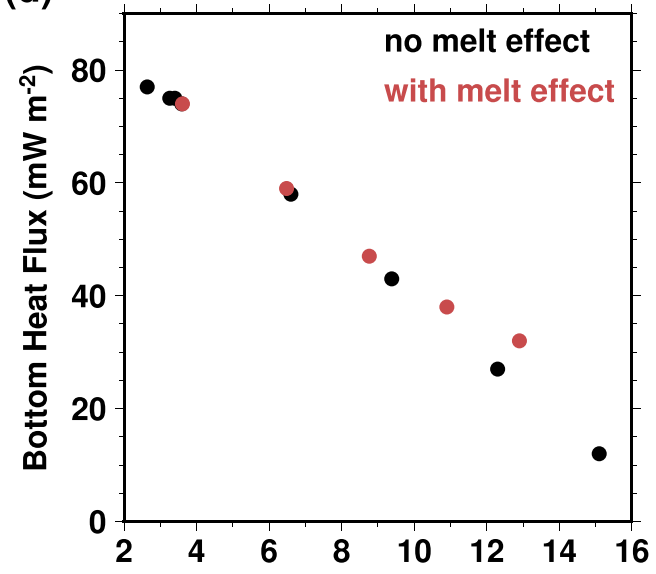

Average Tidal Heating rate $\left(10^{-6} \mathrm{~W} \mathrm{~m}^{-3}\right)$

(e)

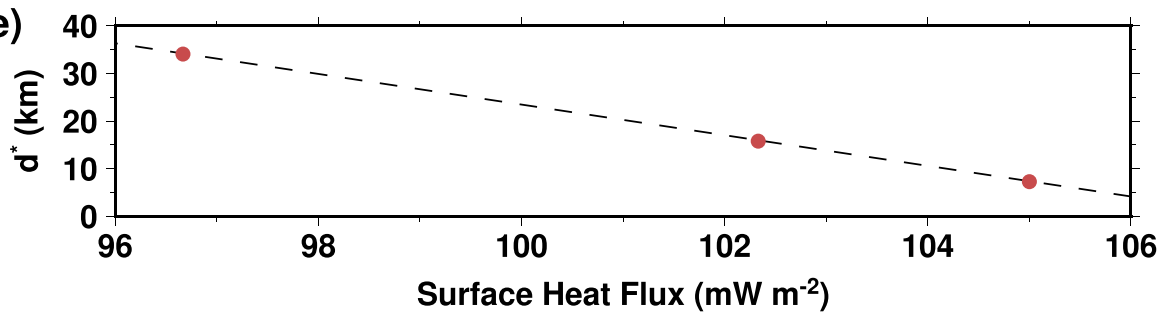

Figure 4. Variations of the $(a, c)$ surface and $(b, d)$ bottom heat flux of the ice layer as a function of the average tidal heating rate. Black symbols correspond to results obtained without the effects of melting, while red symbols are the results including the effects of melting following the approach proposed by Choblet et al. (2017). We assume the case of Europa for a pure water case with a viscosity contrast of $10^{5}$ and an ice layer thickness of (a, b) $15.8 \mathrm{~km}$ (squares), $34.1 \mathrm{~km}$ (triangles) or (c, d) $7.3 \mathrm{~km}$ (circles). When the tidal heating rate is high enough, the surface heat flux reaches a plateau. The value of the plateau is reported in panel (e) as a function of the ice layer thickness $\left(d^{*}\right)$. The dashed line represents a linear fit of the data.

heat flux remains constant (Figure 4c). In this specific case, the volume covered by cold downwellings is larger but decreases with increasing tidal heating rate. The ability of cold downwellings to transport heat and cool down the base of the system is therefore substantially reduced, as illustrated with the increasing interior temperature (Table 3), which in turn induces a decrease of the bottom heat flux. By contrast, thicker ice shells are characterized by more vigorous and chaotic convection, so that cold downwellings are sinking with a much higher speed and are only slightly heated by tidal heating. Nevertheless, when tidal heating is strong enough, and owing to the observed saturation of the surface heat flux, a given value of the surface 

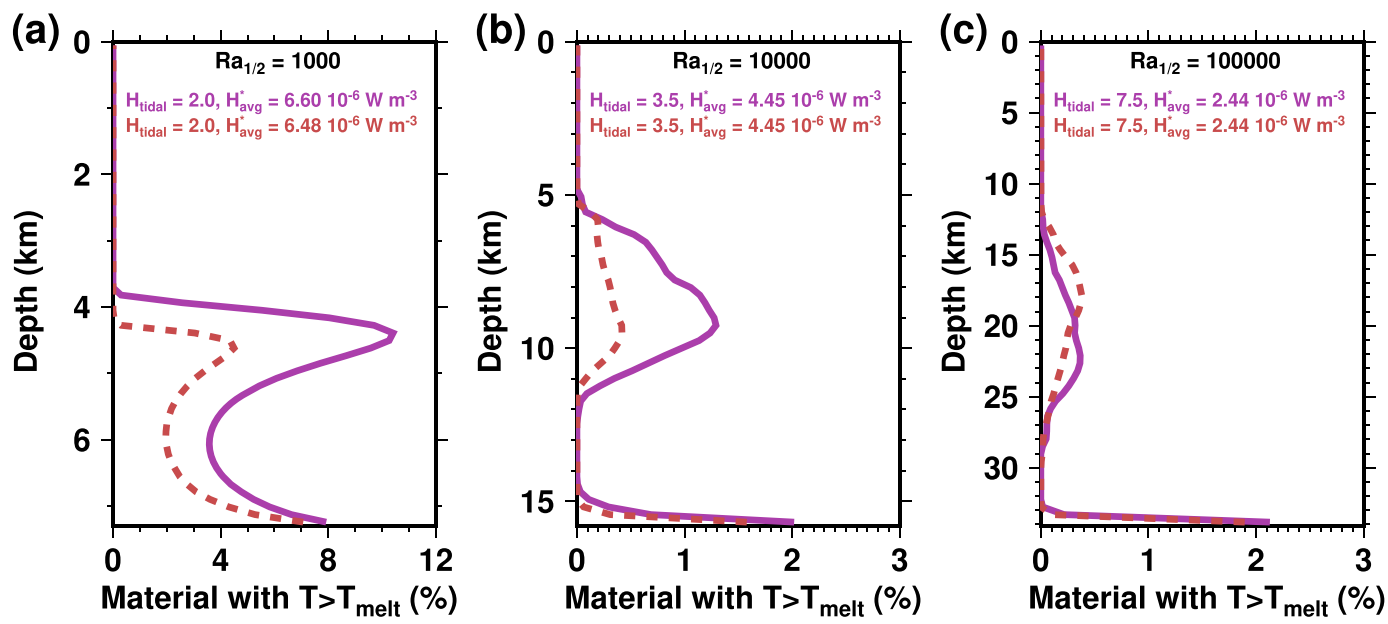

Figure 5. Proportion of material above the melting temperature as a function of depth for $\eta=10^{5}$. A proportion of $100 \%$ and $0 \%$ means the material is fully above the melting temperature or fully solid, respectively. Calculations are done using the properties of Europa given in Table 2, and the thickness of the ice layer is constrained from the assumed value of $R a_{1 / 2}$. Here, the subsurface ocean is composed of pure water, and the melting temperature is given by equation (7). Purple lines correspond to cases without the effects of melting, while red lines include the effects of melting following the approach of Choblet et al. (2017).

heat flux may be associated to a given value of the ice shell thickness (Figure 4e). This means that, in this specific regime, the estimate of the surface heat flux provides a robust estimate of the ice shell thickness. Another interesting consequence is that a fairly large bottom heat flux can be maintained even for large tidal heating rates, thanks to melting and melt extraction.

We also found that melting rates obtained with this approach (Table 3) are quite high. For instance, tidal heating may be able to melt the equivalent of the volume of the ice shell in about 25 million years for a thickness of $34.1 \mathrm{~km}$ (assuming $H_{\text {avg }}^{*}=2.9410^{-6} \mathrm{~W} / \mathrm{m}^{3}$ ) and in only 5 million years for a thickness of $15.8 \mathrm{~km}$ (assuming $H_{\text {avg }}^{*}=6.4710^{-6} \mathrm{~W} / \mathrm{m}^{3}$ ), while increasing $H_{\text {avg }}^{*}$ by $10 \%$ tends to roughly double the proportion of the ice layer that melts every million of years. Note that these tidal heating rates correspond to the upper bound of what is expected for the current state of Europa. It remains unclear whether such heating rates are reached in practice (section 4). Furthermore, the melting profiles (Figure 5) indicate that the shape of the melting region is equivalent to what was obtained for the corresponding case without melt extraction, so that isolated melt pockets are still generated only for a thickness of 15.8 and $34.1 \mathrm{~km}$. Therefore all the conclusions drawn using the previous approach are confirmed when introducing the latent heat effect of melting in the heat budget, that is, (i) melting is expected to occur for a large range of parameters, (ii) proportion of melting increases very sharply with the tidal heating rate, and (iii) isolated melt pockets are only generated for certain values of the ice layer thickness. As a consequence, the comparison between the two sets of numerical simulations shows that the first set of numerical simulations can be applied to any icy satellites to investigate the conditions required to generate melting and the location where the first melt appears. It is, however, necessary to consider a more complex modeling to assess the proportion of melting and the fate of this melt.

\subsection{Influence of Contaminants in the Hydrosphere: Example of $\mathrm{MgSO}_{4}$}

In previous sections, we considered the case of a hydrosphere composed of pure water. However, as indicated by Galileo observations of induced magnetic fields for Europa (Khurana et al., 1998), Ganymede (Kivelson et al., 2002), and Callisto (Khurana et al., 1998), subsurface oceans may be composed of a mixture of water and impurities. The nature of these impurities may be different for each body and includes, for instance, magnesium sulfate $\left(\mathrm{MgSO}_{4}\right)$, ammonia $\left(\mathrm{NH}_{3}\right)$, or sodium chloride $(\mathrm{NaCl})$. Vance et al. (2018) combined the available constraints to build potential interior structure models for different icy satellites. In particular, a subsurface ocean including $10 \mathrm{wt} \%$ of $\mathrm{MgSO}_{4}$ or $3.5 \mathrm{wt} \%$ of $\mathrm{NaCl}$ was considered for Europa.

These impurities have special properties that play a key role in the history of icy bodies and the generation of melts within their ice shell. More specifically, antifreeze compounds remain mostly in the liquid water ocean thus decreasing its freezing temperature as the ice layer thickens. Therefore, in presence of impurities, the 
temperature at the bottom of the ice layer is set by the melting temperature of the water plus antifreeze mixture, while the melting temperature within the ice layer is still imposed by the melting temperature of pure water (equation (7)). As a consequence, subsurface oceans may be colder, and generating melting within the ice layer would become more difficult as the proportion of antifreeze compounds increases. Furthermore, for a fixed amount of antifreeze compounds in the hydrosphere, their concentration in the subsurface ocean increases as the thickness of the ice layer increases. This mechanism has been used to explain the long-term preservation of subsurface oceans (e.g., Deschamps \& Sotin, 2001; Kamata, 2018; Spohn \& Schubert, 2003). It also indicates that melting should be easier for thin ice layers or equivalently thick subsurface oceans.

Given the potential variability of hydrosphere compositions, it is necessary to follow a simple approach allowing to quantify the possible effects of impurities in general. Following the model used by Vance et al. (2018), we choose to consider a mixture of water with $10 \mathrm{wt} \%$ of $\mathrm{MgSO}_{4}$ as a representative solution. The melting temperature of this mixture can be calculated using the framework proposed by Vance et al. (2014). Here, for the sake of simplicity, we use a fit of these results:

$$
T_{\text {melt }}^{*}=271.7-0.07831 p^{*}-0.000134 p^{* 2},
$$

which is valid for pressure up to $200 \mathrm{MPa}$. For this mixture, we report in Table 1 the amplitude and minimum depth of melting for all our simulations. Our results indicate a moderate impact of impurities on the occurrence of melting. More specifically, the main effect of $\mathrm{MgSO}_{4}$ is to induce a colder bottom temperature, so that larger amounts of tidal heating are required to reach the melting point. It should, however, be noted that the generation of melting is suppressed when considering a very large amount of $\mathrm{MgSO}_{4}(>30 \mathrm{wt} \%)$. Furthermore, the distribution of melting is becoming more localized (i.e., melting areas are not connected to the subsurface ocean) when the amount of impurities in the ocean increases. Due to impurities, the temperature at the bottom of the ice layer is lower than the melting temperature at that depth, and melting is thus impossible close to the interface.

These results show that the occurrence of melting depends on the proportion of antifreeze compounds, which is probably different for each icy satellite. Increasing the amount of impurities makes the generation of melting more difficult and, when melting is possible, requires a larger tidal heating rate. When considering a likely composition for the present subsurface ocean of Europa, the presence of contaminants does not influence significantly the occurrence of melting. This conclusion might, however, depend on the nature of the contaminant considered and the structure of the hydrosphere. For example, including $5 \mathrm{wt} \%$ of $\mathrm{NH}_{3}$ has similar effects to the addition of $30 \mathrm{wt} \%$ of $\mathrm{MgSO}_{4}$, that is, to suppress almost entirely the melt generation. Although these concentrations appear to be higher than available estimates, it may be possible to reach this concentration of $\mathrm{NH}_{3}$ in some icy bodies when their ice layer becomes sufficiently thick. From a more general perspective, it is important to remember the double effect of increasing the ice layer thickness: (i) Dynamical properties of the ice layer imply that the generation of melt close to the surface becomes more difficult as the ice layer thickens (Figure 3) and (ii) the relative amount of antifreeze increases with the thickening of the layer, which further prevents the generation of melt. As a consequence, if impurities are present in the subsurface ocean, the occurrence of melting is favored for thin layers and large tidal heating rates.

\section{Discussion: The Thermal State of Europa's Ice Shell}

Based on our simulations describing melting in ice shells of icy bodies, it is tempting to provide a reappraisal of Europa's thermal state. This, however, implies several major assumptions: (1) The temperature dependence of the thermal conductivity can be neglected, (2) the effect of melt on dynamics does not impact significantly the heat budget, and (3) a global steady-state for heat transfer is reached. We now discuss each of these assumptions and their relevance in the case of Europa.

Thermal conductivity of ice Ih is characterized by a relatively important temperature dependence. Although some slight discrepancies exist between experimental works (Andersson \& Suga, 1994; Hobbs, 1974), they all suggest a thermal conductivity that varies as the inverse of the temperature. Accounting explicitly for the effects of temperature-dependent thermal conductivity would require appropriate numerical simulations, which is beyond the scope of this paper. Some qualitative remarks can, however, be made. First, because thermal conductivity decreases with temperature, the conductivity would be smaller at the bottom of the ice shell than at its top $\left(\sim 2.2-2.5\right.$ and $\sim 5.6-6.8 \mathrm{~W} \cdot \mathrm{m}^{-1} \cdot \mathrm{K}^{-1}$, respectively, for Europa), thus limiting the bottom 
heat flux and increasing the ratio between the internal and bottom heat sources. Second, Tobie et al. (2003) have pointed out that the thickness of the stagnant lid would be larger (by about 50\% for Europa) in the case of temperature-dependent thermal conductivity, further reducing the heat transfer toward the surface. Overall, these two effects would favor the occurrence of melting within the shell, while the minimal depths for melting reported above for a constant thermal conductivity (i.e., $\sim 5 \mathrm{~km}$ or less) could be in practice 1.5 times larger.

Melting affects in various ways the evolution of icy shells. While we have estimated its impact on the heat budget, we neglected the influence of melting on density and viscosity. This may be an issue because the presence of melt would reduce the buoyancy of hot plumes and lower the amount of tidal heat generated. In particular, when these effects are included, Tobie et al. (2003) have shown that it may lead to an episodic behavior of plumes, thus oscillatory melt generation. Interestingly, some of our numerical simulations are somewhat similar to cases conducted by Tobie et al. (2003). For these cases, heat fluxes and average amount of melt are equivalent in the two sets of models. The main difference between these models is the evolution of the average melting rate. While the average melting proportion is exhibiting oscillation in Tobie et al. (2003), it is almost constant in our models. Overall, in the parameter range appropriate for Europa's ice shell, the global heat budget should be mostly unaffected by changes in buoyancy and viscosity induced by the presence of melt.

By essence, our simulations assume that a thermal equilibrium is reached not only in the ice shell but also, more generally, in the hydrosphere. This implicitly applies to phases in Europa's evolution for which the thickness of the ice shell evolves on timescales sufficiently large to neglect their effect on the convective dynamics. In the case where the effect of melting is either unaccounted for or negligible in the heat budget, this implies that the heat extracted by thermal convection from the bottom interface $\left(\phi_{b o t}^{*}\right)$ is equal to the heat flux originating from the deep interior that crosses the ice-ocean interface $\left(\phi_{o}^{*}\right)$. By contrast, when the effect of melt is significant, $\phi_{o}^{*}$ includes both the convective heat flux $\left(\phi_{b o t}^{*}\right)$ and the (negative) heat flux related to the extraction of melt from the ice shell into the ocean $\left(-P_{\text {melt }}^{*} / A_{i / o}\right)$, where $P_{\text {melt }}^{*}$ is the global heat loss caused by melting (see equation (9)) and $A_{i / o}$ the surface area of the ice/ocean interface. The implicit assumption of a heat equilibrium in the hydrosphere requires (e.g., Hussmann \& Spohn, 2004)

$$
\phi_{b o t}^{*}-\phi_{o}^{*}=P_{m e l t}^{*} / A_{i / o}=\rho^{*} L^{*} \dot{d}^{*}
$$

with $L^{*}$ the latent heat of fusion and $\dot{d}^{*}=a_{\text {melt }}^{*} d^{*}$ the time derivative of the ice shell thickness (see equation (10) introducing $a_{m e l t}^{*} d^{*}$ ). This condition implies, in turn, that the mass loss caused by the ice melting is compensated by a global crystallization of the ocean $\left(\dot{d}^{*}>0\right)$ induced by a value of $\phi_{o}^{*}$ smaller than $\phi_{b o t}^{*}$. Again, in practice, this assumption could be violated in periods of fast melting of the ice shell or fast crystallization of the ocean. Using a much simpler description for ice convection than the models presented here, but taking into account the whole satellite structure and its orbital evolution in resonance with Io and Ganymede, Hussmann and Spohn (2004) showed that both phases of quasi-steady evolution and phases with runaway melting/freezing of the ice shell should be envisioned during Europa's long-term thermo-orbital history. If tidal power is mostly dissipated in Europa's silicate mantle, then the ice shell evolution is mostly quasi-steady and our assumption is valid. If tidal dissipation in the ice shell is predominant, oscillatory phases for eccentricity and tidal heating might occur during a nonnegligible fraction of Europa's history.

Under these assumptions, we report in Figure 6a the values of $\phi_{o}^{*}$ calculated using equation (12) for all our simulations (Table 3). Note that, while the ranges of values displayed in Figure 6 for $d^{*}$ and $H_{\text {avg }}^{*}$ correspond to those observed in our simulations, the continuous surfaces are an interpolation constructed from only a small number of points (Table 3), potentially introducing large uncertainties. Figure 6 may then be used to guide the analysis but not to make accurate predictions for Europa. In that sense, we have identified various regimes whose estimated boundary are indicated by the thick black curves: "NM" refers to the no-melting regime obtained for cases where the tidal heating rate (measured by $H_{\text {avg }}^{*}$ ) is not sufficient to induce melting within the ice shell (or at rates that hardly affect heat transfer); "SM" indicates the saturated melting regime corresponding to cases displayed in Figure 4, where red symbols present an asymptotic behavior in which increasing the rate of tidal heating results only in a marginal increase (respectively decrease) of the surface (respectively bottom) heat flux; "SM+OH" refers to the ocean heating case, tidal heating is large enough to induce a net heating of the ocean ( $\phi_{o}^{*}$ is negative), while solid-state convection within the ice still extracts heat from the ocean $\left(\phi_{b o t}^{*}\right.$ remains positive). Such cases correspond to phases during which the shell 


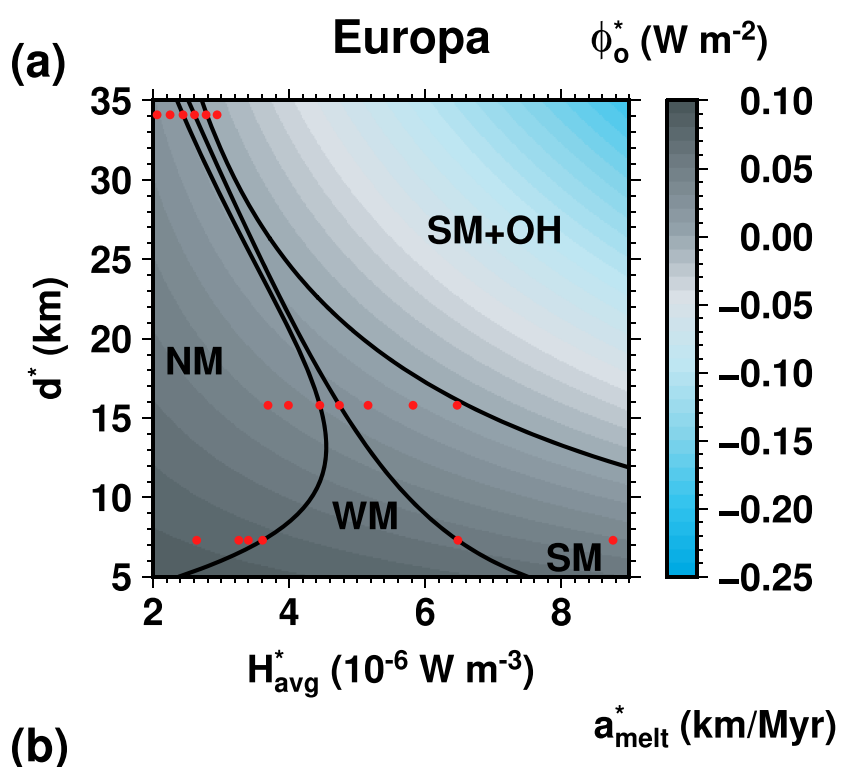

(b)

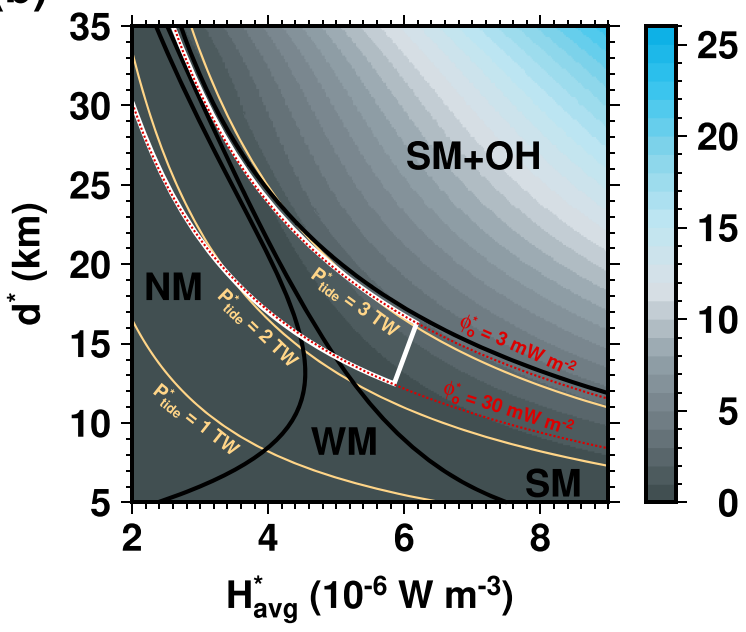

Figure 6. (a) Heat flux from the deep interior of Europa that crosses the ice-ocean interface $\left(\phi_{0}^{*}\right)$ and (b) melting rate of the ice shell $\left(a_{m e l t}^{*}\right)$ as a function of the ice shell thickness $\left(d^{*}\right)$ and average tidal heating rate $\left(H_{\text {avg }}^{*}\right)$ for a viscosity contrast $\Delta \eta=10^{5}$. We also indicate the different regimes observed: "NM" is no melting, "SM" is saturated melting, "WM" is intermediate weak melting, and "OH" is ocean heating (see text for more details). Note that the figures are built from an interpolation based on a small number of points reported in (a) with the red circles (see Table 3 for the full set). The two dashed red lines in (b) correspond to the curves with isovalue $\phi_{o}^{*}=3 \mathrm{~mW} / \mathrm{m}^{2}$ and $\phi_{o}^{*}=30 \mathrm{~mW} / \mathrm{m}^{2}$, while the white quadrangle highlights the plausible parameter values for Europa (see text). The solid orange lines indicate the $\left(d^{*}, H_{\text {avg }}^{*}\right)$ conditions corresponding to a power provided by tidal heating $\left(P_{\text {tide }}^{*}\right)$ of 1,2 , or $3 \mathrm{TW}$.

thickness globally decreases; "WM" denotes an intermediate "weak" melting regime where internal melting within the ice shell affects convective heat transfer but does not result yet in the asymptotic "SM" regime.

A complementary outcome of our simulations is presented in Figure 6b, showing the rate of melting $a_{\text {melt }}^{*}$ induced by tidal heating within the ice shell expressed as the equivalent thickness of ice consumed by this process (Table 3). Values globally reflect that larger amounts of melt are produced for both larger shell thicknesses $d^{*}$ and tidal heating rates $H_{\text {avg }}^{*}$. The $\mathrm{SM}+\mathrm{OH}$ regime is obtained for typical melting rates larger than $5 \mathrm{~km} / \mathrm{Myr}$ for the thinner shells but would occur as soon as $a_{\text {melt }}^{*}$ exceeds $1 \mathrm{~km} / \mathrm{Myr}$ for shells thicker than $25 \mathrm{~km}$. We note that, within our steady-state assumption, global crystallization rates might easily exceed the transient variations of ice shell thickness predicted by the thermo-orbital models of Hussmann and Spohn (2004). This suggests that the present analysis may be valid even during fast oscillatory evolution of Europa. 
To obtain more conclusive results, it is helpful to restrict the plausible parameter space by using available constraints. A first restriction involves admissible values of the net heat flux from the ocean $\phi_{o}^{*}$. The radiogenic heat content of Europa's rocky mantle should provide a few hundreds of gigawatts, probably between 100 and 500 GW (Tobie et al., 2003; Hussmann et al., 2010). Depending on the thermal state of this mantle, additional heating might occur through tidal dissipation: Albeit uncertain, this contribution may be in the same order than the radiogenic heat source (Běhounková et al., 2019). Moreover, dissipation of heat within the liquid ocean might occur either by ohmic dissipation (e.g., Gissinger \& Petitdemange, 2019) or tidal dissipation (e.g., Tyler, 2014). It is, however, unlikely that they constitute a dominant contribution throughout most of Europa's history. Overall, if secular cooling/heating of the deep interior (ocean, rocky mantle, and core) is neglected, a conservative range for $\phi_{o}^{*}$ could be $3-30 \mathrm{~mW} / \mathrm{m}^{2}$ (dashed red curves in Figure $6 \mathrm{~b}$ ). Another limiting factor is the average tidal heating rate. Tobie et al. (2003) predict maximum rates $H_{\max }^{*}$ varying between $2 \times 10^{-6}$ and $8 \times 10^{-6} \mathrm{~W} / \mathrm{m}^{3}$ depending on the location. For Europa, such volumetric rates are not expected to vary significantly with the ice shell thickness. A peak heating of $H_{\max }^{*}=8 \times 10^{-6} \mathrm{~W} / \mathrm{m}^{3}$ would translate for $H_{\text {avg }}^{*}$ into a value smaller than $6 \times 10^{-6} \mathrm{~W} / \mathrm{m}^{3}$ for $d^{*}=15.8 \mathrm{~km}$ (Table 1). Together with the constraint supplied by a probable range for $\phi_{o}^{*}$ (dashed red lines on Figure $6 \mathrm{~b}$ ), we thus consider that reasonable thicknesses for the ice shell exceed $15 \mathrm{~km}$. Under the two restrictions discussed above, the quadrangle bounded by white lines in Figure $6 \mathrm{~b}$ represents the plausible fraction of the parameter space. It involves the three regimes (NM, WM, and SM) and melting rates that are lower than $2 \mathrm{~km} / \mathrm{Myr}$. We note, however, that the total amount of tidal heat $\left(P_{\text {tide }}^{*}\right)$ dissipated in the ice shell for such probable cases is large: The lower limit $\left(\phi_{o}^{*}=30 \mathrm{~mW} / \mathrm{m}^{2}\right)$ roughly corresponds to $P_{\text {tide }}^{*}=2 \mathrm{TW}$. The upper limit $\left(\phi_{o}^{*}=3 \mathrm{~mW} / \mathrm{m}^{2}\right)$ corresponds approximately to $P_{\text {tide }}^{*}=3 \mathrm{TW}$. Such values are in excess to what is predicted by thermo-orbital evolution models (typically 1 TW) when dissipation is allowed within Europa's ice shell only (Hussmann \& Spohn, 2004). If a significant fraction of tidal dissipation also occurs within Europa's rocky mantle, such values could be even harder to achieve. One way to solve this potential discrepancy is by considering a much thicker ice shell ( $\sim 50-90 \mathrm{~km})$. In that case, a significant extrapolation of our results suggests that a typical tidal power of $1 \mathrm{TW}$ could be obtained for a reasonable range of $\phi_{o}^{*}$. For instance, $\phi_{o}^{*}=15 \mathrm{~mW} / \mathrm{m}^{2}$ corresponds to a thickness of $\sim 70-80 \mathrm{~km}$.

\section{Conclusion}

We have conducted a series of 70 numerical simulations designed to study thermal convection within the outer shell of icy satellites. Our models include a strongly temperature-dependent viscosity (with $\Delta \eta$ between $10^{4}$ and $10^{6}$ ), and tidal heating following the approach of Tobie et al. (2003), in which the local tidal heating rate is a function of the viscosity, hence of temperature. With this approach, numerical simulations are not bound to a specific body and can thus be applied to various cases. We then applied our results to the production of melting in Europa and found that isolated melt pockets can be generated fairly close to the surface $(\sim 5-7 \mathrm{~km})$ provided that the ice layer thickness remains modest $\left(15 \leq d^{*} \leq 35 \mathrm{~km}\right)$. Another important finding is that the volume fraction of the temperature field above the melting point increases sharply with increasing tidal heating rates $\left(H_{a v g}\right)$. Because these simulations did not include the effects of melting on the heat budget, we ran 15 additional simulations incorporating this complexity. The comparison between the two sets of simulations shows that models neglecting the effect of melting are still appropriate to investigate the conditions under which melting is generated and the location where the first melt appears. Our numerical simulations can therefore be applied to various icy bodies to assess the presence of melting in their outer ice shells. The second set of numerical simulations is useful to investigate cases with fractions of the temperature field above the melting point. In that case, we found that the surface heat flux remains constant above a certain value of the tidal heating rate, corresponding to the threshold value for the generation of a nonnegligible amount of melt (saturated melting regime). This indicates that if tidal heating is large enough to generate melting, the surface heat flux is a good proxy for the thickness of the ice shell.

Building on these results, we proposed a tentative melting regime diagram for Europa's ice shell. For a given shell thickness, increasing the amount of internal tidal heating leads from a state with no melting (NM) to the saturated melting (SM) regime. For the largest values envisioned, convection extracts heat from the ocean to the ice shell (the bottom heat flux related to convection is positive), but a net ocean heating is expected if melt generated in the ice shell percolates downward, back to the ocean. Reasonable estimates of the heat produced in Europa's deep interior at present, together with plausible values of tidal heating within the ice shell, favor shell thicknesses larger than $15-20 \mathrm{~km}$. We note that the global amount of tidal heat dissipated 
Acknowledgments

We thank two anonymous reviewers for their thorough and constructive comments that helped us to produce a much improved paper. We also thank L. Montesi for his editorial handling of the manuscript. The interpretation of the results benefited from discussions with Chloé Michaut and Shunichi Kamata. This research was funded by the Ministry of Science and Technology of Taiwan (MOST) Grants 106-2116-M-001-014 and 107-2116-M-001-010 and by JSPS KAKENHI Grant JP19F19023. Numerical computations were performed on IESAS Linux Cluster. The data used for generating the figures are available for academic purposes (Vilella, 2020). The code used in this work is not publicly available but was thoroughly described in Tackley (2008). The modifications made to the code are detailed in Choblet et al. (2017). within the ice shell (typically 2-3 TW) is larger than the one proposed by thermo-orbital evolution models during quasi-steady stages. Such a discrepancy could result from a simplified treatment of heat transfer in thermo-orbital models. Alternatively, one possible solution reconciling our simulations (albeit requiring a significant extrapolation of the trends suggested by our results) with such models is to consider ice shells as thick as $\sim 80 \mathrm{~km}$. Interestingly, for such conditions, Europa's ice shell may be in the saturated melting regime, in which case one may expect the surface heat flux to be fairly homogeneous.

\section{References}

Allu Peddinti, D., \& McNamara, A. K. (2015). Material transport across Europa's ice shell. Geophysical Research Letters, 42, 4288-4293. https://doi.org/10.1002/2015GL063950

Allu Peddinti, D., \& McNamara, A. K. (2019). Dynamical investigation of a thickening ice-shell: Implications for the icy moon Europa. Icarus, 329, 251-269. https://doi.org/10.1016/j.icarus.2019.03.037

Anderson, J. D., Lau, E. L., Sjogren, W. L., Schubert, G., \& Moore, W. B. (1997). Europa's differentiated internal structure: Inferences from two Galileo encounters. Science, 276, 1236-1239. https://doi.org/10.1126/science.276.5316.1236

Anderson, J. D., Schubert, G., Jacobson, R. A., Lau, E. L., Moore, W. B., \& Sjogren, W. L. (1998). Europa's differentiated internal structure: Inferences from four Galileo encounters. Science, 281, 2019-2022. https://doi.org/10.1126/science.281.5385.2019

Andersson, O., \& Suga, H. (1994). Thermal conductivity of low-density amorphous ice. Solid State Communications, 91, 985-988. https:// doi.org/10.1016/0038-1098(94)90438-3

Arakawa, M., \& Maeno, N. (1994). Effective viscosity of partially melted ice in the ammonia-water system. Geophysical Research Letters, 21, 1515-1518. https://doi.org/10.1029/94GL01041

Běhounková, M., Tobie, G., Choblet, G., Kervazo, M., \& Dumoulin, C. (2019). Sustainability of the melting in the silicate mantle of Europa. abstract EPSC.

Běhounková, M., Tobie, G., Choblet, G., \& Čadek, O. (2010). Coupling mantle convection and tidal dissipation: Applications to Enceladus and Earth-like planets. Journal of Geophysical Research, 115, E09011. https://doi.org/10.1029/2009JE003564

Běhounková, M., Tobie, G., Choblet, G., \& Čadek, O. (2013). Impact of tidal heating on the onset of convection in Enceladus's ice shell. Icarus, 226, 898-904. https://doi.org/10.1016/j.icarus.2013.06.033

Barr, A. C., \& Hammond, N. P. (2015). A common origin for ridge-and-trough terrain on icy satellites by sluggish lid convection. Physics of the Earth and Planetary Interiors, 249, 18-27. https://doi.org/10.1016/j.pepi.2015.09.009

Boussinesq, J. (1903). Théorie analytique de la chaleur mise en harmonie avec la thermodynamique et avec la théorie mécanique de la lumière, Tome II. Paris: Gauthier-Villars.

Čadek, O., Běhounková, M., Tobie, G., \& Choblet, G. (2017). Viscoelastic relaxation of Enceladus's ice shell. Icarus, 291, 31-35. https://doi. org/10.1016/j.icarus.2017.03.011

Čadek, O., Souček, O., Běhounková, M., Choblet, G., Tobie, G., \& Hron, J. (2019). Long-term stability of Enceladus' uneven ice shell. Icarus, 319, 476-484. https://doi.org/10.1016/j.icarus.2018.10.003

Chizhov, V. E. (1993). Thermodynamic properties and thermal equations of the state of high-pressure ice phases. Journal of Applied Mechanics and Technical Physics, 34, 253-262. https://doi.org/10.1007/BF00852521

Choblet, G. (2005). Modelling thermal convection with large viscosity gradients in one block of the 'cubed sphere'. Journal of Computational Physics, 205, 269-291. https://doi.org/10.1016/j.jcp.2004.11.005

Choblet, G., Tobie, G., Sotin, C., Běhounková, M., Čadek, O., Postberg, F., \& Souček, O. (2017). Powering prolonged hydrothermal activity inside Enceladus. Nature Astronomy, 1, 841-847. https://doi.org/10.1038/s41550-017-0289-8

Choblet, G., Tobie, G., Sotin, C., Kalousová, K., \& Grasset, O. (2017). Heat transport in the high-pressure ice mantle of large icy moons Icarus, 285, 256-262. https://doi.org/10.1016/j.icarus.2016.12.002

Davaille, A., \& Jaupart, C. (1993). Transient high-Rayleigh-number thermal convection with large viscosity variation. Journal of Fluid Mechanics, 253, 141-166. https://doi.org/10.1017/S0022112093001740

Deschamps, F., \& Sotin, C. (2001). Thermal convection in the outer shell of large icy satellites. Journal of Geophysical Research, 106, 5107-5121. https://doi.org/10.1029/2000JE001253

Dombard, A. J., Patterson, G. W., Lederer, A. P., \& Prockter, L. M. (2013). Flanking fractures and the formation of double ridges on Europa. Icarus, 223, 74-81. https://doi.org/10.1016/j.icarus.2012.11.021

Durham, W. B., Prieto-Ballesteros, O., Goldsby, D. L., \& Kargel, J. S. (2010). Rheological and thermal properties of icy materials. Space Science Reviews, 153, 273-298. https://doi.org/10.1007/s11214-009-9619-1

Elachi, C., Wall, S., Allison, M., Anderson, Y., Boehmer, R., Callahan, P., et al. (2005). Cassini radar views the surface of Titan. Science, 308, 970-974. https://doi.org/10.1126/science.1109919

Gaidos, E. J., \& Nimmo, F. (2000). Tectonics and water on Europa. Nature, 405, 637. https://doi.org/10.1038/35015170

Gissinger, C., \& Petitdemange, L. (2019). A magnetically driven equatorial jet in Europa's ocean. Nature Astronomy, 3, 401-407. https:// doi.org/10.1038/s41550-019-0713-3

Goldsby, D. L., \& Kohlstedt, D. L. (2001). Superplastic deformation of ice: Experimental observations. Journal of Geophysical Research, 106, 11,017-11,030. https://doi.org/10.1029/2000JB900336

Han, L., \& Showman, A. P. (2010). Coupled convection and tidal dissipation in Europa's ice shell. Icarus, 207, 834-844. https://doi.org/10. 1016/j.icarus.2009.12.028

Han, L., \& Showman, A. P. (2011). Coupled convection and tidal dissipation in Europa's ice shell using non-Newtonian grain-size-sensitive (GSS) creep rheology. Icarus, 212, 262-267. https://doi.org/10.1016/j.icarus.2010.11.034

Hansen, C. J., Esposito, L., Stewart, A. I. F., Colwell, J., Hendrix, A., Pryor, W., \& West, R. (2006). Enceladus' water vapor plume. Science, 311, 1422-1425. https://doi.org/10.1126/science.1121254

Harel, L., Dumoulin, C., Choblet, G., Tobie, G., \& Besserer, J. (2020). Scaling of heat transfer in stagnant lid convection for the outer shell of icy moons: Influence of rheology. Icarus, 338, 113448. https://doi.org/10.1016/j.icarus.2019.113448

Hobbs, P. V. (1974). Ice physics. New York: Oxford University Press.

Hogenboom, D. L., Kargel, J. S., Consolmagno, T. C., Holden, G. J., Lee, L., \& Buyyounouski, M. (1997). The ammonia-Water system and the chemical differentiation of icy satellites. Icarus, 128, 171-180. https://doi.org/10.1006/icar.1997.5705

Howell, S. H., \& Pappalardo, R. T. (2018). Band formation and ocean-surface interaction on Europa and Ganymede. Geochemistry Geophysics, Geosystems, 45, 4701-4709. https://doi.org/10.1029/2018GL077594 
Howell, S. H., \& Pappalardo, R. T. (2019). Can Earth-like plate tectonics occur in ocean world ice shells? Icarus, 322, 69-79. https://doi. org/10.1016/j.icarus.2019.01.011

Hussmann, H., Choblet, G., Lainey, V., Matson, D. L., Sotin, C., Tobie, G., \& Van Hoolst, T. (2010). Implications of rotation, orbital states, energy sources, and heat transport for internal processes in icy satellites. Space Science Reviews, 153, 314-348. https://doi.org/10.1007/ s11214-010-9636-0

Hussmann, H., \& Spohn, T. (2004). Thermal-orbital evolution of Io and Europa. Icarus, 171, 391-410. https://doi.org/10.1016/j.icarus.2004 05.020

Jankowski, D. G., \& Squyres, S. W. (1988). Solid-state ice volcanism on the satellites of Uranus. Science, 242, 1322-1325. https://doi.org/10. 1126/science.241.4871.1322

Jia, X., Kivelson, M. G., Khurana, K. K., \& Kurth, W. S. (2018). Evidence of a plume on Europa from Galileo magnetic and plasma wave signatures. Nature Astronomy, 2, 459-464. https://doi.org/10.1038/s41550-018-0450-z

Johnson, B. C., Sheppard, R. Y., Pascuzzo, A. C., Fisher, E. A., \& Wiggins, S. E. (2017). Porosity and salt content determine if subduction can occur in Europa's ice shell. Journal of Geophysical Research: Planets, 122, 2765-2778. https://doi.org/10.1002/2017JE005370

Kalousová, K., Souček, O., Tobie, G., Choblet, G., \& Čadek, O. (2014). Ice melting and downward transport of meltwater by two-phase flow in Europa's ice shell. Journal of Geophysical Research: Planets, 119, 532-549. https://doi.org/10.1002/2013JE004563

Kalousová, K., Souček, O., Tobie, G., Choblet, G., \& Čadek, O. (2016). Water generation and transport below Europa's strike-slip faults. Journal of Geophysical Research: Planets, 121, 2444-2462. https://doi.org/10.1002/2016JE005188

Kamata, S. (2018). One-dimensional convective thermal evolution calculation using a modified mixing length theory: Application to Saturnian icy satellites. Journal of Geophysical Research: Planets, 123, 93-112. https://doi.org/10.1002/2017JE005404

Kamata, S., Matsuyama, I., \& Nimmo, F. (2015). Tidal resonance in icy satellites with subsurface oceans. Journal of Geophysical Research: Planets, 120, 1528-1542. https://doi.org/10.1002/2015JE004821

Kargel, J. S. (1995). Cryovolcanism on the icy satellites. Earth, Moon, and Planets, 67, 101-113. https://doi.org/10.1007/BF00613296

Kattenhorn, S. A., \& Prockter, L. M. (2014). Evidence for subduction in the ice shell of Europa. Nature Geoscience, 7, 762-767. https://doi. org/10.1038/ngeo2245

Khurana, K. K., Kivelson, M. G., Stevenson, D. J., Schubert, G., Russell, C. T., Walker, R. J., \& Polanskey, C. (1998). Induced magnetic fields as evidence for subsurface oceans in Europa and Callisto. Nature, 395, 777-780. https://doi.org/10.1038/27394

Kirk, R. L., \& Stevenson, D. J. (1987). Thermal evolution of a differentiated Ganymede and implications for surface features. Icarus, 69, 91-134. https://doi.org/10.1016/0019-1035(87)90009-1

Kivelson, M. G., Khurana, K. K., \& Volwerk, M. (2002). The permanent and inductive magnetic moments of Ganymede. Icarus, 157, 507-522. https://doi.org/10.1006/icar.2002.6834

Kuskov, O. L., \& Kronrod, V. A. (2005). Internal structure of Europa and Callisto. Icarus, 177, 550-569. https://doi.org/10.1016/j.icarus 2005.04.014

Lesage, E., Massol, H., \& Schmidt, F. (2020). Cryomagma ascent on Europa. Icarus, 335. https://doi.org/10.1016/j.icarus.2019.07.003

Lopes, R. M. C., Mitchell, K. L., Stofan, E. R., Lunine, J. I., Lorenz, R., Paganelli, F., et al. (2007). Cryovolcanic features on Titan's surface as revealed by the Cassini Titan Radar Mapper. Icarus, 186, 395-412. https://doi.org/10.1016/j.icarus.2006.09.006

Lopes, R. M. C., Kirk, R. L., Mitchell, K. L., LeGall, A., Barnes, J. W., Hayes, A., \& Malaska, M. J. (2013). Cryovolcanism on Titan: New results from Cassini RADAR and VIMS. Journal of Geophysical Research: Planets, 118, 416-435. https://doi.org/10.1002/jgre.20062

Lorenz, R. D., Stiles, B. W., Kirk, R. L., Allison, M. D., del Marmo, P. P., Iess, L., \& Hensley, S. (2008). Titan's rotation reveals an internal ocean and changing zonal winds. Science, 319, 1649-1651. https://doi.org/10.1126/science.1151639

Manga, M., \& Michaut, C. (2017). Formation of lenticulae on Europa by saucer-shaped sills. Icarus, 286, 261-269. https://doi.org/10.1016/ j.icarus.2016.10.009

Melosh, H. J., \& Janes, D. M. (1989). Ice volcanism on Ariel. Science, 245, 195-196. https://doi.org/10.1126/science.245.4914.195-a

Mével, L., \& Mercier, E. (2005). Resorption process in Astypalaea Linea extensive region(Europa). Planetary and Space Science, 53, 771-779. https://doi.org/10.1016/j.pss.2004.12.005

Michaut, C., \& Manga, M. (2014). Domes, pits, and small chaos on Europa produced by water sills. Journal of Geophysical Research: Planets, 119, 550-573. https://doi.org/10.1002/2013JE004558

Mitri, G., \& Showman, A. P. (2005). Convective-conductive transitions and sensitivity of a convecting ice shell to perturbations in heat flux and tidal-heating rate: Implications for Europa. Icarus, 177, 447-460. https://doi.org/10.1016/j.icarus.2005.03.019

Mitri, G., Showman, A. P., Lunine, J. I., \& Lopes, R. M. C. (2008). Resurfacing of Titan by ammonia-water cryomagma. Icarus, 196, $216-224$. https://doi.org/10.1016/j.icarus.2008.02.024

Moresi, L. N., \& Solomatov, V. S. (1995). Numerical investigation of 2D convection with extremely large viscosity variations. Physics of Fluids, 7, 2154-2162. https://doi.org/10.1063/1.868465

Nimmo, F., \& Gaidos, E. (2002). Strike-slip motion and double ridge formation on Europa. Journal of Geophysical Research, 107, 5-1-5-8. https://doi.org/10.1029/2000JE001476

Nimmo, F., \& Manga, M. (2002). Causes, characteristics and consequences of convective diapirism on Europa. Geophysical Research Letters, 29(23), 2109. https://doi.org/10.1029/2002GL015754

Nimmo, F., \& Pappalardo, R. T. (2016). Ocean worlds in the outer solar system. Journal of Geophysical Research: Planets, 121, 1378-1399. https://doi.org/10.1002/2016JE005081

Ogawa, M., Schubert, G., \& Zebib, A. (1991). Numerical simulations of three-dimensional thermal convection in a fluid with strongly temperature-dependent viscosity. Journal of Fluid Mechanics, 233, 299-328. https://doi.org/10.1017/S0022112091000496

Ojakangas, G. W., \& Stevenson, D. J. (1989). Thermal state of an ice shell on Europa. Icarus, 81, 220-241. https://doi.org/10.1016/ 0019-1035(89)90052-3

Pappalardo, R. T., Reynolds, S. J., \& Greeley, R. (1997). Extensional tilt blocks on Miranda: Evidence for an upwelling origin of Arden Corona. Journal of Geophysical Research, 102, 13,369-13,379. https://doi.org/10.1029/97JE00802

Porco, C. C., Helfenstein, P., Thomas, P. C., Ingersoll, A. P., Wisdom, J., West, R., \& Squyres, S. (2006). Cassini observes the active south pole of Enceladus. Science, 311, 1393-1401. https://doi.org/10.1126/science.1123013

Reese, C. C., Solomatov, V. S., \& Moresi, L. N. (1999). Non-Newtonian stagnant lid convection and magmatic resurfacing on Venus. Icarus, 139, 67-80. https://doi.org/10.1006/icar.1999.6088

Roberts, J. H., \& Nimmo, F. (2008). Tidal heating and the long-term stability of a subsurface ocean on Enceladus. Icarus, 194, 675-689. https://doi.org/10.1016/j.icarus.2007.11.010

Roth, L., Saur, J., Retherford, K. D., Strobel, D. F., Feldman, P. D., McGrath, M. A., \& Nimmo, F. (2014). Transient water vapor at Europa's south pole. Science, 343, 171-174. https://doi.org/10.1126/science.1247051 
Schenk, P. M. (1991). Fluid volcanism on Miranda and Ariel: Flow morphology and composition. Journal of Geophysical Research, 96, 1887-1906. https://doi.org/10.1029/90JB01604

Schenk, P. M., McKinnon, W. B., Gwynn, D., \& Moore, J. M. (2001). Flooding of Ganymede's bright terrains by low-viscosity water-ice lavas. Nature, 410, 57-60. https://doi.org/10.1038/35065027

Schmidt, B. E., Blankenship, D. D., Patterson, G. W., \& Schenk, P. M. (2011). Active formation of 'chaos terrain' over shallow subsurface water on Europa. Nature, 479, 502-505. https://doi.org/10.1038/nature10608

Showman, A. P., \& Han, L. (2004). Numerical simulations of convection in Europa's ice shell: Implications for surface features. Journal of Geophysical Research, 109, E01010. https://doi.org/10.1029/2003JE002103

Smith, B. A., Soderblom, L. A., Banfield, D., Barnet, C., Basilevksy, A. T., Beebe, R. F., et al. (1989). Voyager 2 at Neptune: Imaging science results. Science, 246, 1422-1449. https://doi.org/10.1126/science.246.4936.1422

Sotin, C., Head, J. W. III, \& Tobie, G. (2002). Europa: Tidal heating of upwelling thermal plumes and the origin of lenticulae and chaos melting. Geophysical Research Letters, 29(8), 1233. https://doi.org/10.1029/2001GL013844

Sotin, C., \& Tobie, G. (2004). Internal structure and dynamics of the large icy satellites. Comptes Rendus Physique, 5, 769-780. https://doi. org/10.1016/j.crhy.2004.08.001

Sparks, W. B., Schmidt, B. E., McGrath, M. A., Hand, K. P., Spencer, J. R., Cracraft, M., \& Deustua, S. E. (2017). Active cryovolcanism on Europa? The Astrophysical Journal Letters, 839, L18. https://doi.org/10.3847/2041-8213/aa67f8

Spencer, J. R., Pearl, J. C., Segura, M., Flasar, F. M., Mamoutkine, A., Romani, P., \& Lopes, R. M. C. (2006). Cassini encounters Enceladus: Background and the discovery of a south polar hot spot. Science, 311, 1401-1405. https://doi.org/10.1126/science.1121661

Spencer, J. R., Tamppari, L. K., Martin, T. Z., \& Travis, L. D. (1999). Temperatures on Europa from Galileo Photopolarimeter-Radiometer: Nighttime thermal anomalies. Science, 284, 1514-1516. https://doi.org/10.1126/science.284.5419.1514

Spohn, T., \& Schubert, G. (2003). Oceans in the icy Galilean satellites of Jupiter? Icarus, 161, 456-467. https://doi.org/10.1016/ S0019-1035(02)00048-910.1016/S0019-1035(02)00048-9

Tackley, P. J. (2008). Modelling compressible mantle convection with large viscosity contrasts in a three-dimensional spherical shell using the yin-yang grid. Physics of the Earth and Planetary Interiors, 171, 7-18. https://doi.org/10.1016/j.pepi.2008.08.005

Tobie, G., Choblet, G., \& Sotin, C. (2003). Tidally heated convection: Constraints on Europa's ice shell thickness. Journal of Geophysical Research, 108(E11), 5124. https://doi.org/10.1029/2003JE002099

Tobie, G., Grasset, O., Lunine, J. I., Mocquet, A., \& Sotin, C. (2005). Titan's internal structure inferred from a coupled thermal-orbital model. Icarus, 175, 496-502. https://doi.org/10.1016/j.icarus.2004.12.007

Trumbo, S. K., Brown, M. E., \& Butler, B. J. (2018). ALMA thermal observations of Europa. The Astronomical Journal, 156, 161-167. https:// doi.org/10.3847/1538-3881/aada87

Tyler, R. (2014). Comparative estimates of the heat generated by ocean tides on icy satellites in the outer solar system. Icarus, 243, 358-385. https://doi.org/10.1016/j.icarus.2014.08.037

Vance, S., Bouffard, M., Choukroun, M., \& Sotin, C. (2014). Ganymede's internal structure including thermodynamics of magnesium sulfate oceans in contact with ice. Planetary and Space Science, 96, 62-70. https://doi.org/10.1016/j.pss.2014.03.011

Vance, S. D., Panning, M. P., Stähler, S., Cammarano, F., Bills, B. G., Tobie, G., \& Banerdt, B. (2018). Geophysical investigations of habitability in ice-covered ocean worlds. Journal of Geophysical Research: Planets, 123, 180-205. https://doi.org/10.1002/2017JE005341

Vilella, K. (2020). Tidally heated convection and the occurrence of melting in icy satellites: Application to Europa. Mendeley Data, version 1. https://doi.org/10.17632/4wg5crstkm.1 